\title{
Investigating the nature of the extended structure around the Herbig star RCrA using integral field and high-resolution spectroscopy
}

E. Rigliaco ${ }^{1}$, R. Gratton ${ }^{1}$, D. Mesa ${ }^{1}$, V. D’Orazi ${ }^{1}$, M. Bonnefoy ${ }^{2}$, J. M. Alcalà ${ }^{3}$, S. Antoniucci ${ }^{4}$, F. Bacciotti ${ }^{5}$, M. Dima ${ }^{1}$, B. Nisini ${ }^{4}$, L. Podio ${ }^{5}$, M. Barbieri ${ }^{6}$, R. Claudi ${ }^{1}$, S. Desidera ${ }^{1}$, A. Garufi ${ }^{5}$, E. Hugot ${ }^{7}$, M. Janson ${ }^{8,9}$, M. Langlois ${ }^{10}$, E. L. Rickman ${ }^{11}$, E. Sissa ${ }^{1}$, M. Ubeira Gabellini ${ }^{12}$, G. van der Plas ${ }^{2}$, A. Zurlo ${ }^{13,14,8}$, Y. Magnard ${ }^{15}$, D. Perret ${ }^{16}$, R. Roelfsema ${ }^{17}$, and L. Weber ${ }^{18}$

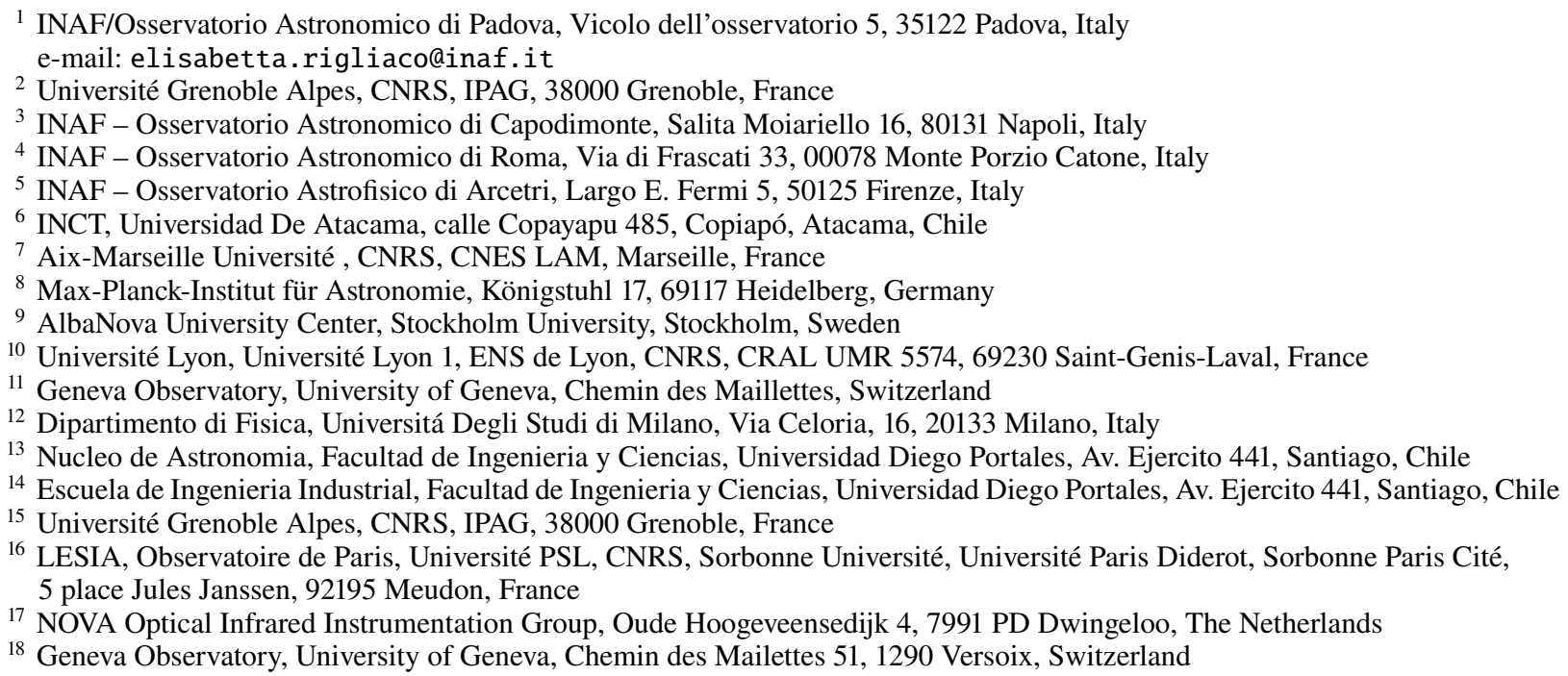

Received 16 September 2019 / Accepted 28 September 2019

\section{ABSTRACT}

\begin{abstract}
Context. We present a detailed analysis of the extended structure detected around the young and close-by Herbig Ae/Be star R CrA. This is a young triple system with an intermediate mass central binary whose separation is of the order of a few tens of the radii of the individual components, and an M-star companion at about 30 au.

Aims. Our aim is to understand the nature of the extended structure by means of combining integral-field and high-resolution spectroscopy.

Methods. We conducted the analysis based on FEROS archival optical spectroscopy data and adaptive optics images and integral-field spectra obtained with SINFONI and SPHERE at the VLT.

Results. The observations reveal a complex extended structure that is composed of at least two components: a non-uniform wide cavity whose walls are detected in continuum emission up to $400 \mathrm{au}$, and a collimated wiggling-jet detected in the emission lines of helium and hydrogen. Moreover, the presence of [Fe II] emission projected close to the cavity walls suggests the presence of a slower moving wind, most likely a disk wind. The multiple components of the optical forbidden lines also indicate the presence of a high-velocity jet co-existing with a slow wind. We constructed a geometrical model of the collimated jet flowing within the cavity using intensity and velocity maps, finding that its wiggling is consistent with the orbital period of the central binary. The cavity and the jet do not share the same position angle, suggesting that the jet is itself experiencing a precession motion possibly due to the wide M-dwarf companion. Conclusions. We propose a scenario that closely agrees with the general expectation of a magneto-centrifugal-launched jet. These results build upon the extensive studies already conducted on R CrA.
\end{abstract}

Key words. stars: pre-main sequence - protoplanetary disks - Herbig-Haro objects - ISM: jets and outflows ISM: individual objects: $\mathrm{R} \mathrm{CrA}$

\section{Introduction}

Herbig Ae/Be stars (Herbig 1960) are pre-main sequence stars of intermediate mass covering the range between low-mass $\mathrm{T}$ Tauri stars (TTSs), and the embedded massive young stellar objects.
They are considered the high-mass counterparts of pre-main sequence T Tauri stars (Strom et al. 1972; Cohen \& Kuhi 1979; Finkenzeller \& Mundt 1984). These stars, like T Tauri stars, show rich emission-lines spectra, infrared continuum excess and veiled photospheric absorption. The formation of stars in the 
low and intermediate-mass regimes involves accretion disks, and fast collimated outflows and jets. The accretion activity is established from a spectroscopic point of view through the presence of emission lines in the stellar spectrum, in wavelength ranges that span from the ultraviolet to the infrared (e.g. Alcalá et al. 2017; Mendigutía et al. 2012). Jets and outflows in TTSs are also spectroscopically revealed by the analysis of emission lines in their spectrum (e.g., Edwards 2007; Nisini et al. 2018), while there is instead a paucity of detected jets and outflows around intermediate mass Herbig Ae/Be stars (e.g., Grady et al. 2003; Ellerbroek et al. 2014). This is partially due to the shorter time the intermediate mass objects spend in their pre-main sequence phase.

The advent of high-contrast scattered light observations of protoplanetary disks around TTSs and Herbig Ae/Be stars performed with Adaptive Optics techniques using instruments such as the Gemini Planet Imager (GPI: Macintosh et al. 2014) or the Spectro-Polarimetric High-contrast Exoplanet REsearch (SPHERE: Beuzit et al. 2019) has allowed for the investigation of the immediate surroundings of these stars, revealing a wealth of extended structures: concentric rings (e.g. Ginski et al. 2016; Perrot et al. 2016; Feldt et al. 2017), cavities (e.g. Pohl et al. 2017; Ligi et al. 2018), spiral arms (e.g. Maire et al. 2017; Benisty et al. 2017), and asymmetries. ALMA, on the other hand, has allowed for the investigation of the immediate surroundings of younger and more embedded stars, revealing in turn non-axisymmetric features (e.g. van der Marel et al. 2013), multiple narrow rings (e.g. Pérez et al. 2016; Fedele et al. 2017, 2018; Huang et al. 2018). These features are usually associated with circumstellar/protoplanetary disks, but there are a few cases where the images reveal the presence of extended and elongated structures in the jet directions. The analysis of these elongated structures from Herbig stars is important because it allows us to study if the origins of the observed features are similar to T Tauri stars, that is, linked to interaction between magnetic fields and the circumstellar environment (Hubrig et al. 2019). Only a few observations of jets around close-by (less than 200 pc from the Sun) Herbig stars exist. A collimated jet around the Herbig Ae star HD 163296 was detected in Ly $\alpha$ by Devine et al. (2000) and Grady et al. (2000). A bipolar jet is also driven by the Herbig Ae stars MWC480 and HD104237 as shown by Grady et al. (2010, 2004). Recently, Garufi et al. (2019) have revealed, in scattered light, a dip along the jet axis around the intermediate mass star RY Tau that is consistent with an outflow cavity carved in the ambient envelope by the jet and the wind outflow. Further out Herbig stars have also been observed driving jets: extended emissions from both components of the Z CMa system have been revealed by Antoniucci et al. (2016) using the ZIMPOL instrument (Zurich Imaging Polarimeter) of SPHERE at the Very Large Telescope (VLT). A jet and counter-jet were also revealed in LkHa233 using the Hubble Space Telescope data (Melnikov et al. 2008). The paucity of observations of jets around Herbig stars with respect to the higher number of jets observed around T Tauri stars might be either due to an intrinsic abundance of TTSs, or to a shorter timescale of these structures around Herbig stars. In either case, any new observation of jets around Herbig stars is essential to shedding light on the accretion/ejection mechanisms operating on Herbig stars.

$\mathrm{R}$ CrA (HIP 93449) is an ideal target to enlarge the number of Herbig Ae/Be stars where jet-like structures are observed. It is the brightest member of the Coronet Cluster, belonging to the Corona Australis star-forming region, which is one of the nearest and most active regions of ongoing star formation. The Coronet Cluster is highly obscured (Taylor \& Storey 1984), and characterized by high and spatially-variable extinction with $A_{\mathrm{V}}$ up to 45 mag (Neuhäuser \& Forbrich 2008). This star has been extensively studied over the years. Takami et al. (2003) suggested the existence of a companion and of an outflow to explain the positional photo-center displacement observed in spectroastrometric observations both in the blue and red-shifted wing of the spectrally resolved $\mathrm{H} \alpha$-line. The spectral type of $\mathrm{R} \mathrm{CrA} \mathrm{has}$ also been largely debated. In analyzing IRAS data, Bibo et al. (1992) found spectral type $\mathrm{B} 8$, stellar radius of $3.1 R_{\odot}$, bolometric luminosity between $99-166 L_{\odot}$, and mass $3.0 M_{\odot}$. Spectral type A5 and $L_{\text {bol }}=92 L_{\odot}$ were found by Chen et al. (1997), while R CrA was classified as F5 by Hillenbrand et al. (1992), Natta et al. (1993), and Garcia Lopez et al. (2006). R CrA is in a particularly early evolutionary phase (Malfait et al. 1998), because it is still embedded in its dust envelope, whose emission dominates the spectral energy distribution from mid-IR to millimeter wavelengths (Kraus et al. 2009). At optical wavelengths, the star is known to be highly variable, both on long and short time scales (Bellingham \& Rossano 1980). Sissa et al. (2019) found $A_{\mathrm{V}}=5.47 \pm 0.5 \mathrm{mag}$, slightly larger than the value obtained by Bibo et al. (1992) $\left(A_{\mathrm{V}}=4.65 \mathrm{mag}\right)$. R CrA shows indications of active accretion, and various outflow tracers have been reported. For instance, a compact bipolar molecular outflow with an eastwest orientation (Walker et al. 1984; Levreault 1988; Graham 1993), as well as several Herbig-Haro objects (in particular HH 104 A/B), have been associated with R CrA (Hartigan \& Graham 1987; Graham 1993). However, more recent studies (Anderson et al. 1997; Wang et al. 2004) convincingly identified the source IRS 7 as the driving source of these outflows, making a physical association with $\mathrm{R}$ CrA rather unlikely. The outflow was also detected in infra-red (IR) through $\mathrm{CO}$ emission (at $4.6 \mu \mathrm{m}$ ) using astrospectrometry by van der Plas et al. (2015). They notice that the $\mathrm{CO}$ emission is located entirely on one side of the IR continuum emission, and blueshifted by $\sim 10 \mathrm{~km} \mathrm{~s}^{-1}$ with respect to the surrounding Corona Australis molecular cloud. They point out that $\mathrm{CO}$ emission in the $\mathrm{R} \mathrm{CrA} \mathrm{spectrum} \mathrm{is}$ likely due to an outflow, as also suggested from the bow-shocks seen in shocked $\mathrm{H} 2$ emission in the immediate vicinity of R CrA (Kumar et al. 2011). The study of R CrA has gained new momentum in recent years, thanks to high-contrast imaging observations obtained with SPHERE (Mesa et al. 2019) and the NAOS-CONICA at VLT (Cugno et al. 2019). These studies focused their attention on the detection of a stellar companion as close as 19-28 au, and with a mass range between 0.1-1.0 $M_{\odot}$. Moreover, in the Mesa et al. (2019) analysis, the presence of an elongated jet-like structure was pointed out, together with some evidence of a disk seen almost edge-on. Recently, Sissa et al. (2019) analyzed the light curve of the star, and found that the central object of R CrA is a binary with masses of 3.0 and $2.3 M_{\odot}$ for the two components and with a period of approximately 66 days. Together with the discovery of the M-type companion, it makes it a triple system.

In this paper, we analyze images of $\mathrm{R} \mathrm{CrA}$ acquired with SPHERE and the SINFONI integral field spectrograph at the VLT, and an archival optical spectrum from the Fibre-fed Optical Echelle Spectrograph (FEROS) archive. In Sect. 2, we describe the collected data. In Sect. 3, we describe the data analysis. In Sect. 4, we propose a scenario that reconciles all the findings, and in Sect. 5, we summarize and conclude.

\section{Observations and data reduction}

As mentioned in the previous section, several studies at different wavelength ranges have been conducted over the years on $\mathrm{R}$ CrA. The use of high-contrast imagers has made it possible 
Table 1. Log of the observations obtained with SPHERE and SINFONI.

\begin{tabular}{ccccccc}
\hline \hline Date & Instrument & Band & FOV & Spaxel & Spectral resolution & Wavelength coverage \\
\hline $2018-06-19$ & IFS & $Y-H$ & $1.7^{\prime \prime} \times 1.7^{\prime \prime}$ & $7.49 \mathrm{mas}^{2}$ & $\sim 30$ & $0.95-1.65 \mu \mathrm{m}$ \\
$2018-06-19$ & IRDIS & $K 1, K 2$ & $11.0^{\prime \prime} \times 11.0^{\prime \prime}$ & $12.25 \mathrm{mas}^{2}$ & - & $2.11 \mu \mathrm{m}, 2.25 \mu \mathrm{m}$ \\
$2018-09-11$ & SINFONI & $H$ & $0.8^{\prime \prime} \times 0.8^{\prime \prime}$ & $12.5 \mathrm{mas}^{2}$ & $\sim 3000$ & $1.45-1.85 \mu \mathrm{m}$ \\
\hline
\end{tabular}

to shed new light on this interesting source. In this section, we describe the observations of $\mathrm{R}$ CrA acquired in recent years with SPHERE and SINFONI at VLT (summarized in Table 1), and FEROS at the $2.2 \mathrm{~m}$ telescope in La Silla. We focus here on the elongated structure already reported in Mesa et al. (2019), and identified in Fig. 1, analyzing the emission spectrum of the source, and investigating the origin of the emitting lines in light of images of the jet-like structure observed in the SPHERE data.

\subsection{SPHERE data}

R CrA was observed with SPHERE (Beuzit et al. 2019) in four different epochs, in coronagraphic mode, as described in Mesa et al. (2019). In this paper, we use the observation of the night 2018-06-19 as part of the SHINE (SpHere INfrared survey of Exoplanets, Chauvin et al. 2017) guaranteed time observations, since they were taken in better weather conditions and reached deeper contrast with respect to the other sets of observations. The instrument was used in IRDIFS_EXT mode, allowing simultaneous observation with the integral-field spectrometer IFS (Claudi et al. 2008) and with the dual band differential imager and spectrograph IRDIS (Dohlen et al. 2008). In this mode, IFS provides diffraction-limited observations covering the $Y$ and $H$ bands $(0.95-1.65 \mu \mathrm{m})$, with a spectral resolution of $\sim 30$ inside the $1.7^{\prime \prime} \times 1.7^{\prime \prime}$ field of view. The IRDIS sub-system, also diffraction-limited, was set in its dual-band imaging mode (DBI, Vigan et al. 2010) to simultaneously observe with the $K 1$ and $K 2$ filters $(K 1=2.110 \mu \mathrm{m}$ and $K 2=2.251 \mu \mathrm{m}$, width $0.1 \mu \mathrm{m})$ with a $11.0^{\prime \prime} \times 11.0^{\prime \prime}$ field of view. Details on the observing mode and data reduction are reported in Mesa et al. (2019). Data were acquired in pupil stabilized mode, with a sequence of acquisition, while the field of view (FOV) rotated. This allows application of angular differential imaging (ADI, Marois et al. 2006) to reduce the impact of speckle noise: in general, we use an approach based on a principal component analysis (PCA, Soummer et al. 2012) for differential imaging. The IFS data allows a further spectral dimension of the hyper-data cube. The IFS image, obtained using a PCA done along temporal and spectral channels (ASDI) simultaneously, with 10 principal components (Mesa et al. 2015) by Mesa et al. (2019), highlighted the presence of an elongated structure in the north-east direction, as well as the hint of a disk seen almost edge-on in the image. In the following we refer to this "elongated structure", deferring its interpretation to the next sections.

In the present paper, we prefer to use images obtained with a PCA done separately on the different spectral channels (monochromatic PCA) in order to retrieve all the information coming from every channel; this makes it possible to highlight the different wavelengths sampled in the image. In addition, we also used images that are obtained simply subtracting a radial-profile and that are not affected by the self-subtraction of non-uniform imaged structures typical of differential imaging. The median IFS image showing the continuum emission in $Y$ and $H$ band and the most prominent line emission of the helium at $1.083 \mu \mathrm{m}$ is shown in Fig. 1. Figure 2 shows the continuum emission obtained in $K 1$ and $K 2$ with IRDIS, and the subtraction of the $K 1-K 2$ filters.

\subsection{SINFONI data}

A $H$-band image of R CrA was obtained with the AO-fed integral field spectrograph SINFONI (Eisenhauer et al. 2003; Bonnet et al. 2004) operating between 1.45 and $1.85 \mu \mathrm{m}$ with a resolution $R \sim 3000$. The data were collected during the night 2018-09-11 under the program 2101.C-5048(A) (PI.: D. Mesa) with a spatial sampling of $0.0125^{\prime \prime} / \mathrm{pxl} \times 0.0125^{\prime \prime} / \mathrm{pxl}$ for a total field of view of $0.8^{\prime \prime} \times 0.8^{\prime \prime}$. The data reduction is described in Mesa et al. (2019). The phase of the central binary of the SINFONI observation is 0.459 (Sissa et al. 2019), and according to the interpretation of the light curve in that paper, the spectrum of the star should be dominated by the secondary at this epoch.

In Fig. 3, we show the composite line emission images obtained for the $\mathrm{H}, \mathrm{H}_{2}$ and [Fe II] lines detected in the SINFONI data. Green refers to $\mathrm{H}$-emission, blue to $\mathrm{H}_{2}$-emission, and red to [Fe II]-emission. While $\mathrm{H}$ and $\mathrm{H}_{2}$ are almost co-located, the $[\mathrm{Fe}$ II] emission shows a clear offset, being present at the southern edge of the "elongated structure" seen in the continuum image. In the same figure, we identify two separated regions: one indicated as "jet" along the "elongated structure" direction, and the other one indicated as "cavity", which extends along the east direction. These regions were chosen arbitrarily, in a region where the bulk of the line emission is observed. We extracted the spectra in these two regions, and they are shown in Fig. 4. The spectra were obtained after dividing the total intensity of each wavelength for a synthetic spectrum of telluric absorption lines, and after subtracting the median continuum emission. The synthetic spectrum of telluric absorption was computed using the synthetic sky modeler, Telfit (Gullikson et al. 2014), adopting the proper parameters for airmass, pressure, humidity, and temperature. The Brackett series hydrogen recombination lines, as well as $\mathrm{H}_{2}$ emission lines, and [Fe II] lines, are identified in the spectra. A faint signal may be detected in Fig. 4 in the direction of the counter-"elongated structure". This is most likely not a totally "elongated structure"-related signal, but it is contaminated by the typical butterfly pattern in the direction of the wind sometimes observed in high-contrast images (Milli et al. 2017). To assess this hypothesis, we checked the wind direction for the night when the SINFONI observations were taken on the Paranal Ambient Conditions Archive, that is indeed $\sim 140^{\circ}$ (counted clockwise from north), in agreement with the direction of the signal from "elongated structure" and counter-"elongated structure".

\subsection{FEROS data}

We retrieved an optical spectrum of R CrA from the ESO dataproduct Archive. It was acquired in 2009 with FEROS as part of the program 083.A-9013(A) and covers the wavelength range from 3500 to $9200 \AA$ with a resolution of $R \simeq 48000\left(\simeq 6 \mathrm{~km} \mathrm{~s}^{-1}\right)$. 

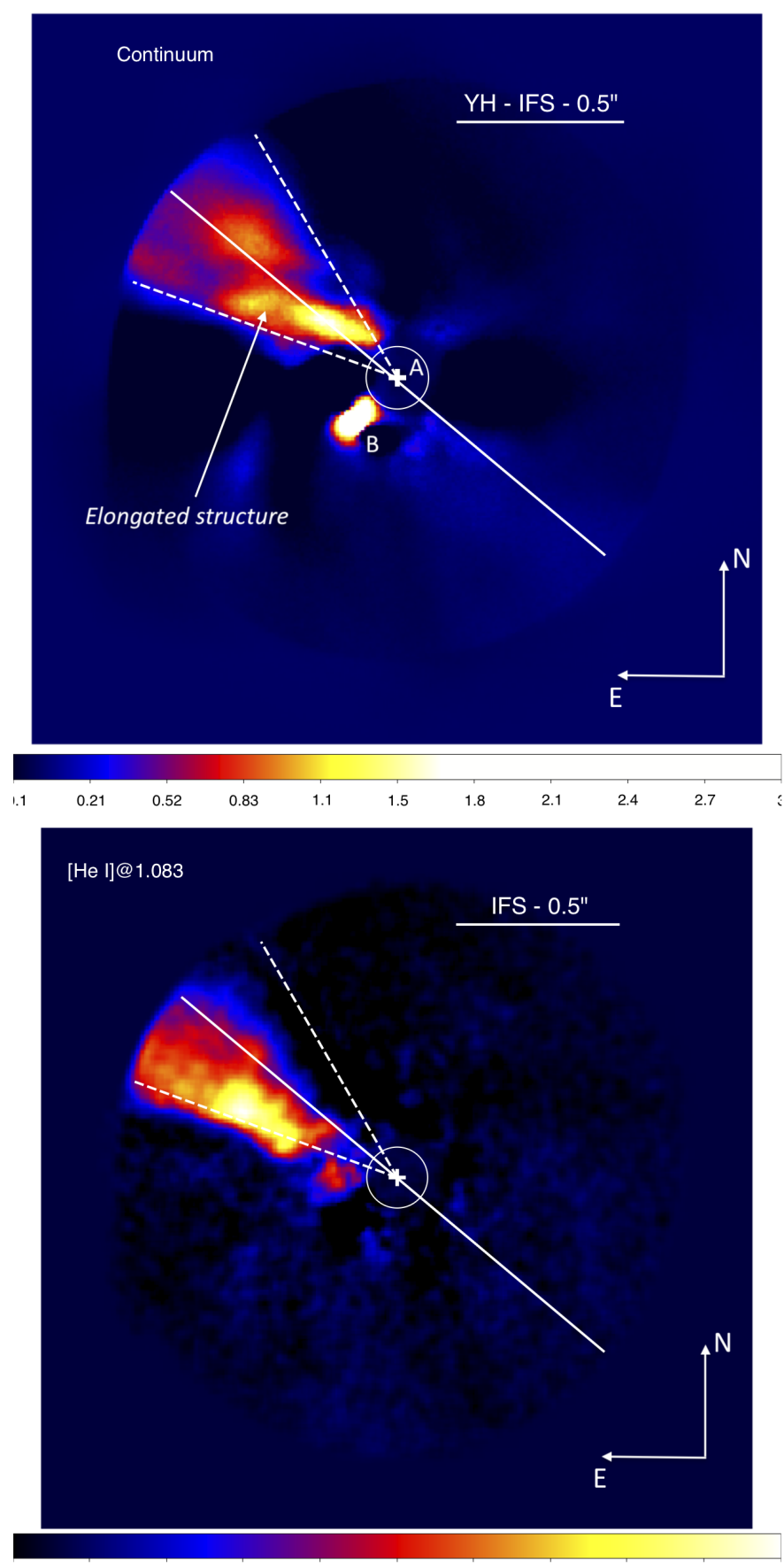

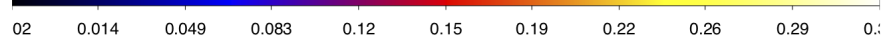

Fig. 1. Top panel: median image obtained using monochromatic PCA with 1 principal component for spectral channel on SPHERE-IFS image, showing the continuum emission. The bright source at about 0.3 arcsec south-east from the center, identified with $\mathrm{B}$, is the M-star companion detected by Mesa et al. (2019), which appears elongated as a consequence of the differential imaging adopted. Solid and dashed lines refer to the median PA and aperture of the "elongated structure" as measured in Sect. 3.2, respectively. Bottom panel: same image as top panel, but only in channels containing helium I at $1.083 \mu \mathrm{m}$ line. The white solid and dashed lines show the PA of the extended structure, as discussed in Sect. 3. The white circle shows the size of the coronagraph used.

The FEROS fiber diameter projected on the sky is $2.7^{\prime \prime}$, and covers radii out to $\sim 200 \mathrm{au}$ at the distance of $\mathrm{R} \mathrm{CrA}$. The spectrum was reduced by performing flat-fielding, wavelength calibration, and barycentric correction. Assuming the $\sim 66$-day period of the
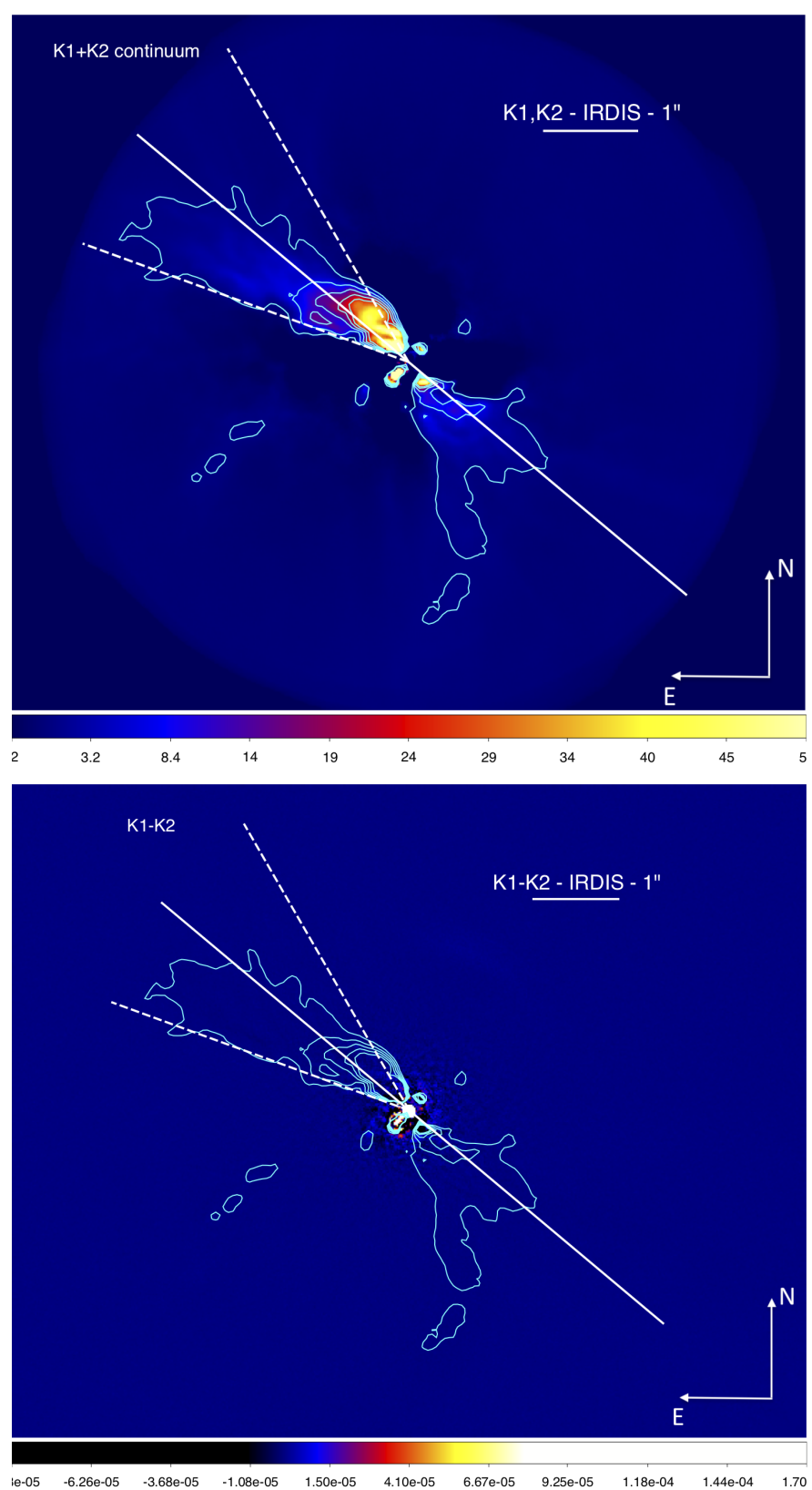

Fig. 2. Top panel: median image obtained using monochromatic PCA with one principal component per spectral channel on SPHERE-IRDIS image, showing continuum emission. Contours are also shown. Bottom panel: subtraction of $K 1-K 2$ filters. No signal is left. For comparison, the contours used in the top panel are also reported. Solid and dashed lines as in Fig. 1.

central binary system (Sissa et al. 2019), at the epoch of the FEROS observation, the spectrum was dominated by the primary star. We identified several emission lines in the spectrum of $\mathrm{R} \mathrm{CrA}$, and we discuss the emission spectrum in the next section.

\section{Data analysis}

The analysis of the images and spectra described in the previous section allows us to investigate the extent, morphology, and physical condition of the gas and dust in the region where the "elongated structure" is observed. The detected lines and continuum probe different components of this complex system and 

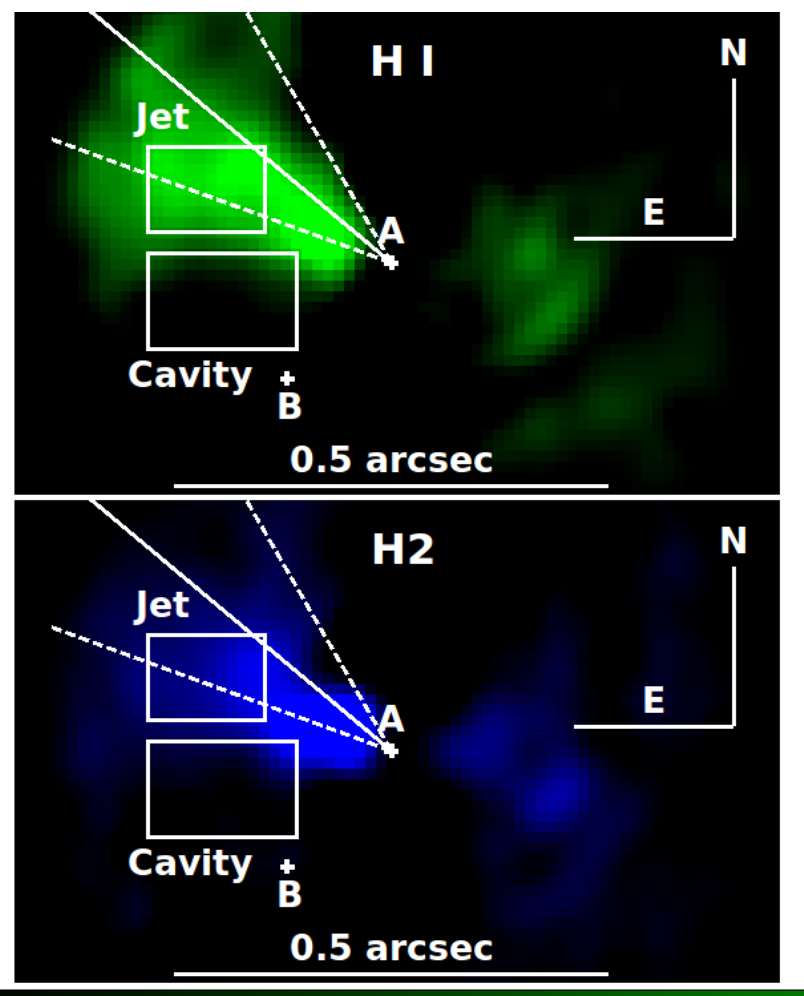

2
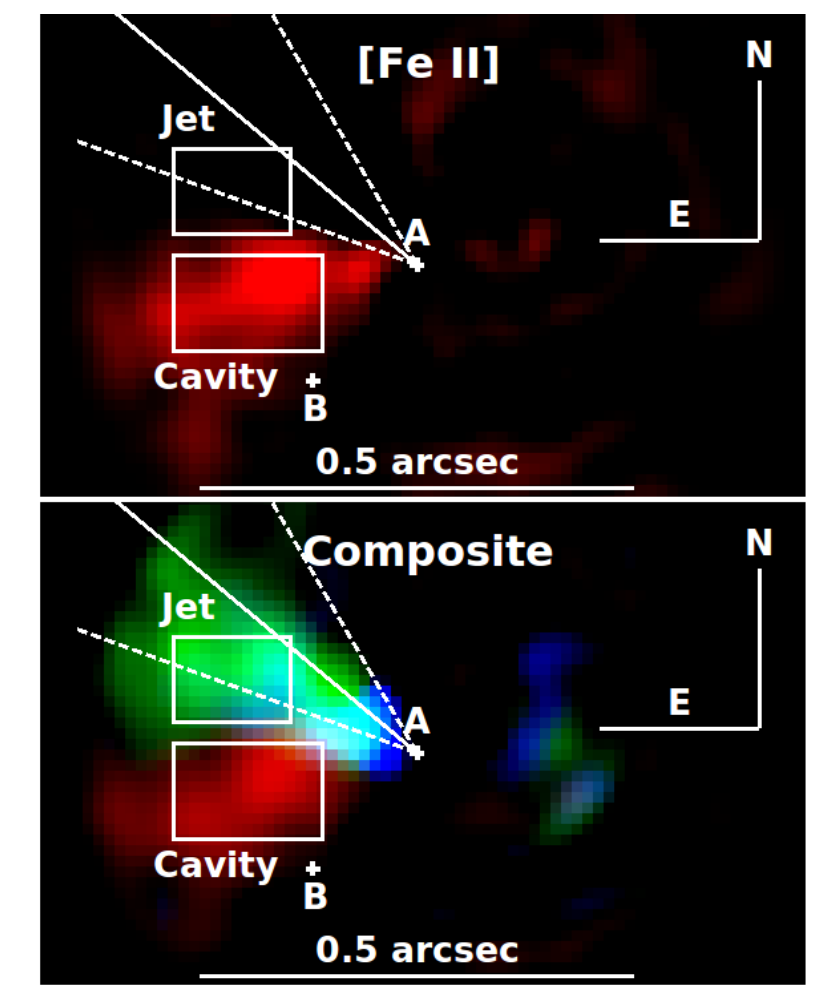

10

12

14

Fig. 3. False-color images obtained from SINFONI data cube in line emission. In green, the summed emission from the hydrogen lines, in blue, the sum of the emission from the $\mathrm{H}_{2}$ lines, and in red, the sum of the emission from the [Fe II] lines. Bottom-right panel: composite image obtained from the sum of the other three panels. The boxes show the regions where we extracted the spectra for the "jet" and "cavity", shown in Fig. 4. Solid and dashed lines as in Fig. 1: they indicate to the "elongated structure" PA and aperture as identified from IFS and IRDIS continuum images.

allow us to derive the properties of the jet, the cavity, and the accreting gas, as explained in the following sections.

\subsection{Gas properties}

The FEROS spectrum covers a wavelength range rich in emission lines that are diagnostic of accretion and outflow activity. Strong, double-peaked, self-absorbed $\mathrm{H} \alpha$ and $\mathrm{H} \beta$ emission lines are observed, together with atomic forbidden lines such as [O I] and [S II].

The ratio between the intensity of the lines of [S II] at 6716 and $6731 \AA$ has been largely used to derive the electron density $n_{\mathrm{e}}$ of the emitting gas, because they have a similar energy of the upper level. The intensity ratio is then sensitive to electron density and almost independent of the temperature (Osterbrock 1989). We used the revised diagnostic diagrams from Proxauf et al. (2014), which give $n_{\mathrm{e}}=7 \times 10^{3} \mathrm{~cm}^{-3}$ for the [S II] $\lambda 6716 / 6731$ line ratio, assuming an electron temperature of $10,000 \mathrm{~K}$. This value is in agreement with the value found from the intensity ratio of the [S II] lines at 4069 and $6731 \AA$ by Hamann (1994).

The high resolution of the FEROS spectrum also allowed us to conduct an analysis on the multiple component of the Oxygen forbidden lines. Emission from [O I] lines in the optical wavelengths is a well established tracer of outflows in T Tauri stars. Several studies of these lines have shown that their emission is often blueshifted and is formed in an outflow whose redshifted part is obscured by the circumstellar disk (e.g. Edwards et al. 1987). Moreover, the [O I] lines observed at medium/highresolution show a profile composed of multiple components: a high-velocity component (HVC) with line peaks shifted by up to a few hundreds $\mathrm{km} \mathrm{s}^{-1}$, and a low-velocity component (LVC) with blueshifts between a few to $\sim 30 \mathrm{~km} \mathrm{~s}^{-1}$. The line components are emitted in physically different regions. The HVC is produced in a fast-moving collimated (micro) jet (e.g. Kwan \& Tademaru 1988; Hartigan et al. 1995), while the LVC has been found to most likely trace disk winds (e.g. Acke et al. 2005; Rigliaco et al. 2013; Natta et al. 2014; Banzatti et al. 2019). In Fig. 5, we show the [O I] $6300 \AA$ and $6363 \AA$ line profiles of $\mathrm{R}$ CrA, and in Table 2, we summarize the forbidden line properties. Both lines clearly show the two components that can be reproduced by Gaussian profiles: in blue the LVC, blueshifted by $\sim 6 \mathrm{~km} \mathrm{~s}^{-1}$, and in green the HVC tracing gas moving at higher velocity. The observed profile is well reproduced by the sum of two Gaussian components (red profile). These features are observed within the 2.7" FEROS fiber, meaning that the gas is distributed within 200 au from the central star. This represents the only solid limit we can put on the region where the HVC form as seen on-sky. More speculatively, if we assume that these lines are emitted from gas in Keplerian orbit around the star, we can use the measured FWHM (see Table 2) to constrain the radius at which they are emitted. Using the sum of the masses of the two components of the central binary system, respectively, $3.0 M_{\odot}$ and a $2.3 M_{\odot}$, we find that under this hypothesis, the [O I] LVC should be emitted at $\sim 2.5$ au from the central star. On the other hand, the LVC can be either magnetically or thermally driven, and in both cases, its origin is within few au from the central star. Following Hartigan et al. (1995), we used the [O I] $6300 \AA$ A HV line luminosity to retrieve an estimate of the mass loss through the jet, to be compared then to the mass accretion rate onto the star. Using Eq. (A8) from Hartigan et al. (1995), assuming 

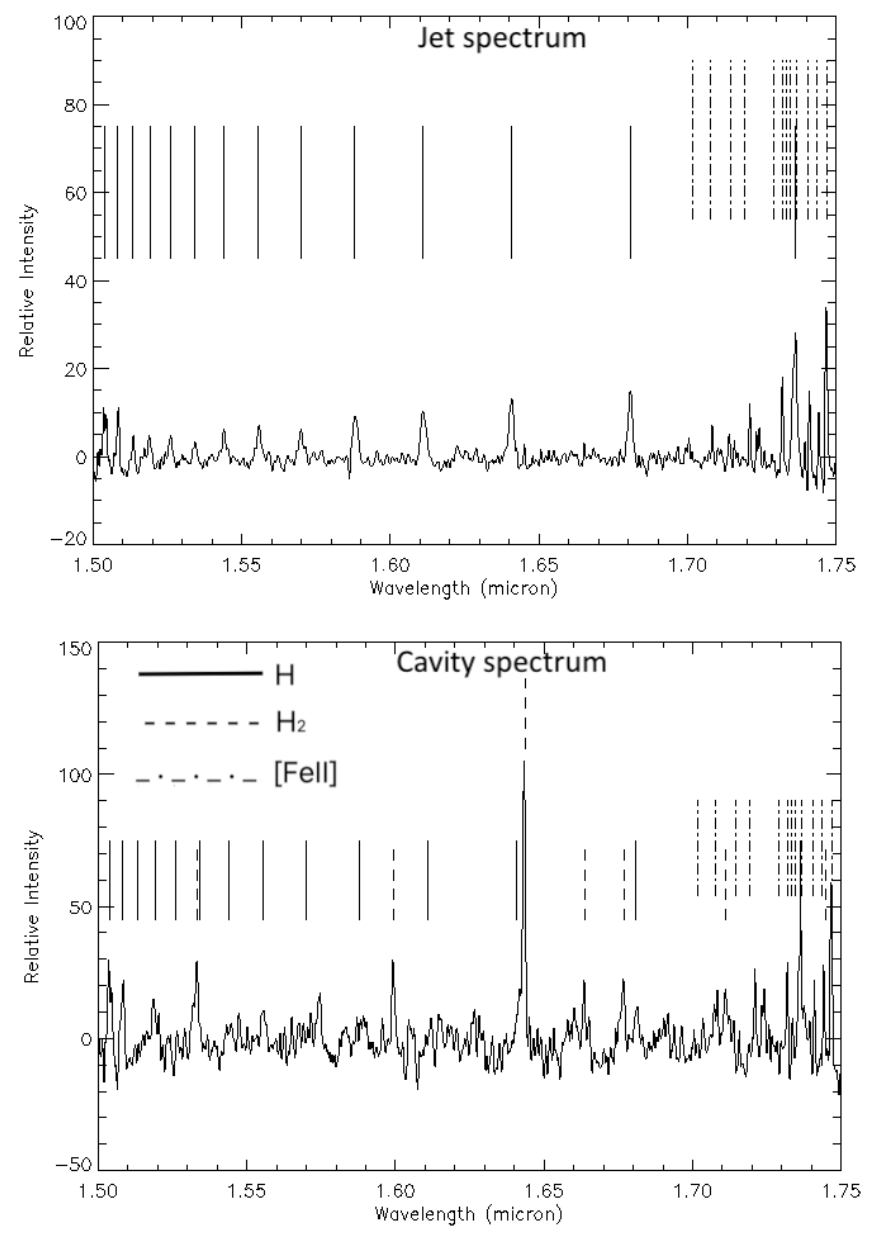

Fig. 4. Spectra from SINFONI data along the elongated structure for both "jet" and the "cavity" components. The locations of the hydrogen recombination lines from the Brackett series are indicated, together with the [Fe II] and $\mathrm{H}_{2}$ lines. The spectra are extracted from the two boxes shown in Fig. 3.

$l_{\perp} \sim 1.0^{\prime \prime}$ as the jet aperture on the plane of the sky (as retrieved from the images), and $v_{\perp}$ the projected velocity of the jet in the sky ( $\sim 60 \mathrm{~km} \mathrm{~s}^{-1}$, as discussed in the next section), we retrieved $\dot{M}_{\text {loss }} \sim 2.8 \times 10^{-7} M_{\odot} \mathrm{yr}^{-1}$. This value is then compared to the accretion rate we retrieved from the $\mathrm{H} \alpha$ and $\mathrm{H} \beta$ lines. We measured the equivalent width (EW) for these lines, and obtained an estimate of the accretion luminosity $L_{\text {acc }}$ of the star using the empirical relationships given by Fairlamb et al. (2017) for Herbig $\mathrm{Ae} / \mathrm{Be}$ stars. To overcome the uncertainty caused by variability of the star, we employed the photometry taken the same night as the FEROS spectrum $\left(V_{\text {mag }}=11.6\right)$ with the All Sky Automated Survey (ASAS, Pojmanski 1997) for these measurements. The measured $\mathrm{EW}_{\mathrm{H} \alpha} \sim 135 \AA$, and $\mathrm{EW}_{\mathrm{H} \beta} \sim 11 \AA$, yield to accretion rate values of $1.6 \times 10^{-6} M_{\odot} \mathrm{yr}^{-1}$ and $5.1 \times 10^{-6} M_{\odot} \mathrm{yr}^{-1}$, respectively. Assuming the average accretion rate is the mean between these two values, we find $\dot{M}_{\text {acc }} \sim 3.3 \times 10^{-6} M_{\odot} \mathrm{yr}^{-1}$. The ratio of the mass accretion to the mass loss rate is $\sim 0.08$, which is consistent with values found for TTSs with prominent stellar jets (e.g., HH34, HH47, HH111 Hartigan et al. 1994, 1995), and it is consistent with a jet that is powered by the accretion onto the central star.

As we mentioned in the previous section, the SINFONI spectrum in the "jet" region covering the near-IR wavelengths range from 1.45 to $1.85 \mu \mathrm{m}$, does show the Brackett series hydrogen
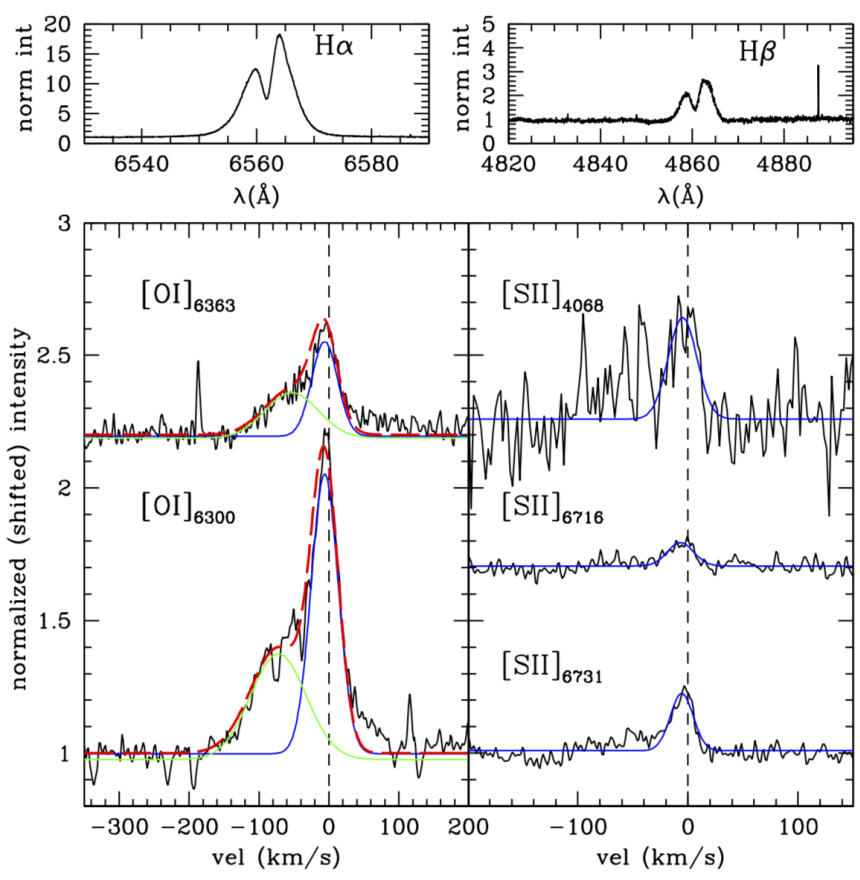

Fig. 5. FEROS spectrum of RCrA. Top panels: regions around $\mathrm{H} \alpha$ and $\mathrm{H} \beta$ lines. Bottom panels: regions around [O I] $\lambda 6716 / 6731$ and [S II] $\lambda 4068 / 6716 / 6731$ forbidden lines. The colored Gaussian profiles in the bottom panels refer to the multiple component deconvolution: in green, the HVC, in blue, the LVC, in red, the sum of the two components.

recombination lines, but there is no detection of [Fe II] lines at $1.53,1.60$ and $1.64 \mu \mathrm{m}$ in this region. Studies of jets from Herbig stars (as also recently found for RY Tau, Garufi et al. 2019) in the optical and in the Near-IR showed that optical jets also emit in the Near-IR [Fe II] lines, unless the density in the jet beam is very low $\left(n_{\mathrm{e}}<10^{3} \mathrm{~cm}^{-3}\right)$. Indeed, owing to their large critical density, these lines need high electron density to be efficiently excited, thus they cannot be used as diagnostics in low-density jets (e.g., Podio et al. 2006). Besides being caused by the low-density gas, the non-detection of the [Fe II] might be due either to the low sensitivity of the $H$-band spectrum, or to the depletion of the iron that is locked into the dust grains (e.g., Podio et al. 2006; Garcia Lopez et al. 2010).

\section{2. "Elongated structure" size}

The SPHERE images of R CrA (IRDIS and IFS) obtained using the monochromatic PCA with 1 principal component are shown in Figs. 1 and 2. In Fig. 6, we show the median spectrum from the IFS data cube. It represents the average signal within the area where we identify the "elongated structure" normalized to the stellar peak. The spectrum is dominated by continuum emission: however, there is some evidence of the contribution due to the emission line of helium at $1.083 \mu \mathrm{m}$, and slight hints of Paschen $\beta$ when the spectrum is normalized to the continuum (Fig. 6, left bottom panel). The steepness of the observed spectrum shows that the "elongated structure" continuum emission has a redder color than the stellar emission. For comparison, we also extracted the spectrum in the region where the almost edge-on disk was pointed out by Mesa et al. (2019) (panels c, d in Fig. 6). We notice that while the reddening of the spectrum in the direction of the disk is in agreement with the spectra extracted for other disks around Herbig stars (e.g., HD 100546, 
Table 2. Observed lines in the FEROS spectrum.

\begin{tabular}{|c|c|c|c|c|c|c|c|}
\hline \multirow[t]{2}{*}{ Element } & \multirow{2}{*}{$\begin{array}{l}\text { Wavelength } \\
\text { (̊) }\end{array}$} & \multicolumn{3}{|c|}{ LVC } & \multicolumn{3}{|c|}{ HVC } \\
\hline & & $\begin{array}{c}v_{\mathrm{c}} \\
\left(\mathrm{km} \mathrm{s}^{-1}\right)\end{array}$ & $\begin{array}{l}F W H M \\
\left(\mathrm{~km} \mathrm{~s}^{-1}\right)\end{array}$ & $\begin{array}{l}\text { EW } \\
(\AA)\end{array}$ & $\begin{array}{c}v_{\mathrm{c}} \\
\left(\mathrm{km} \mathrm{s}^{-1}\right)\end{array}$ & $\begin{array}{l}F W H M \\
\left(\mathrm{~km} \mathrm{~s}^{-1}\right)\end{array}$ & $\begin{array}{l}\text { EW } \\
(\AA)\end{array}$ \\
\hline [O I] & 6300 & $-5.8 \pm 0.2$ & $45.6 \pm 0.7$ & $1.22 \pm 0.01$ & $-71.8 \pm 3.7$ & $94.9 \pm 6.8$ & $0.40 \pm 0.01$ \\
\hline$[\mathrm{O} \mathrm{I}]$ & 6363 & $-6.2 \pm 0.4$ & $43.2 \pm 1.4$ & $0.50 \pm 0.01$ & $-50.4 \pm 7.8$ & $94.7 \pm 14.9$ & $0.16 \pm 0.009$ \\
\hline [S II] & 6731 & $-5.6 \pm 0.6$ & $24.3 \pm 1.7$ & $0.18 \pm 0.01$ & $\ldots$ & $\ldots$ & $\ldots$ \\
\hline [S II] & 6716 & $-7.6 \pm 6.1$ & $28.5 \pm 2.9$ & $0.093 \pm 0.009$ & $\ldots$ & $\ldots$ & $\ldots$ \\
\hline [S II $]$ & 4068 & $-6.8 \pm 4.1$ & $36.0 \pm 12.6$ & $0.28 \pm 0.02$ & $\ldots$ & $\ldots$ & $\ldots$ \\
\hline$[\mathrm{Fe} \mathrm{II}]$ & 7155 & $\ldots$ & $\ldots$ & $\ldots$ & $-35.8 \pm 1.4$ & $129.8 \pm 4.5$ & $0.26 \pm 0.01$ \\
\hline
\end{tabular}
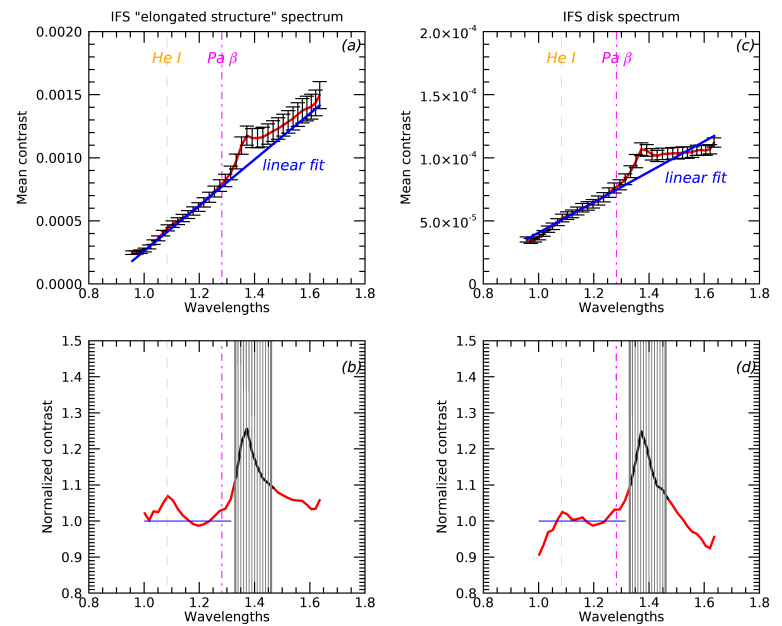

Fig. 6. Spectra extracted from SPHERE-IFS data, once a radial profile, has been subtracted. (a) Spectrum (mean contrast versus wavelengths) extracted in area where "elongated structure" is identified, normalized for stellar peak. The location of the helium and hydrogen $(\mathrm{Pa} \beta)$ line emission are labeled. The blue line shows the linear curve utilized to normalize the spectrum, and reported in the bottom panel. (b) Spectrum normalized to linear fit indicated in top panel. The grey-shaded area shows the telluric band. (c) Same as panel a, but for a region closer to the disk identified by Mesa et al. (2019). (d) Same as panel $b$ for disk.

Sissa et al. 2018 and SAO 206462, Maire et al. 2017), the spectrum along the "elongated structure" appears steeper. We must consider that the circumstellar environment around $\mathrm{RCrA}$ is affected by high extinction (e.g., Bibo et al. 1992; Sissa et al. 2019), and a gradient of extinction within the region itself, hence the reddening of the spectrum might be due to this effect. However, the scattering properties of dusty grains of different size might also play a role in this context.

We analyze the aperture of the "elongated structure". Following on from the analysis of Mesa et al. (2019), we measured the aperture from the IFS continuum image (Fig. 1, top panel). The position angle (PA) of the "elongated structure" spans from $\sim 30^{\circ}$ to $\sim 70^{\circ}$, with a median PA of $\sim 50^{\circ}$. The same PA and "elongated structure" aperture is shown in the IRDIS image, where the contour plot of the "elongated structure" is also shown. The furthest region of the "elongated structure" marked by the contours appear slightly bent toward south. We notice, moreover, that the dust "cavity" region, as identified in the SINFONI image, extends outside the "elongated structure" as identified from the IFS and IRDIS continuum and line images. In particular, the axis of the emission in the dust "cavity" region has a PA of $\sim 100^{\circ}$.
The continuum emission of the "elongated structure" observed in the IFS image, which represents the approaching part to the observer, covers all the IFS field of view, meaning that it extends up to $\sim 120$ au from the central system. From the IRDIS image, the radial extent of the approaching part of the observed "elongated structure" extends up to 2.6" from the central objects (400 au at the R CrA distance), remaining as wide as $\sim 30^{\circ}$ up to $\sim 300$ au. Optically visible jets from TTSs are known to begin with wide (10-30 degree) opening angles close to the source, and are rapidly collimated to within a few degrees in the innermost 50-100 au (Ray et al. 2007; Frank et al. 2014). Wider structures, as the one observed in the continuum emission around $\mathrm{R} \mathrm{CrA}$, might be consistent with shells of ambient gas swept up by the jet bow-shock and a surrounding slower wider-angle component. This wide-angle wind and the swept-up outflow expand more slowly, carving out a cavity, which widens over time into the envelope and the surrounding cloud (Frank et al. 2014).

\subsection{Morphology}

The overall morphology of the observed "elongated structure" as seen in the continuum emission from IFS and IRDIS images appears non-uniform and discontinuous along the radial extent. Moreover, the SINFONI image shows a wiggling pattern in the hydrogen lines. We analyzed the wiggling as a function of the distance from the central binary by focusing both on the emission lines and on the continuum emission. We employed the same method used by Antoniucci et al. (2016): we considered a set of contiguous slices orthogonal to the jet axis and in each slice we fitted the pixel distribution with a Gaussian function in order to obtain the profile peak positions as a function of the distance from the star. We applied this method to the SPHERE-IFS channels containing the He I line at $1.083 \mu \mathrm{m}$, to the SINFONI channels containing the Brackett series lines at 1.555, 1.641, $1.681,1.736 \mu \mathrm{m}$, and to the IFS average continuum emission. The results are shown in Fig. 7, where the plots from the gas components are shown in the top panels, and the continuum emission is shown in the bottom panel. We first notice that the "elongated structure" seen in the gaseous component (both helium and hydrogen) is at a median PA of $\sim 65^{\circ}$, higher than the median PA $\left(\sim 50^{\circ}\right)$ found from the continuum emission, pointing to a misalignment between the "elongated structure" axis seen in the continuum and the gas emission. This misalignment can already be clearly noted in Fig. 1, where the He I emission (bottom panel) does not share the same PA as the continuum emission (top panel). The same happens for the hydrogen, [Fe II] and $\mathrm{H}_{2}$ lines shown in Fig. 3, where the lines are not emitted at the same PA as the continuum in $Y-H$ and $K$ bands from the IFS and IRDIS images. Both helium and hydrogen emissions display a 

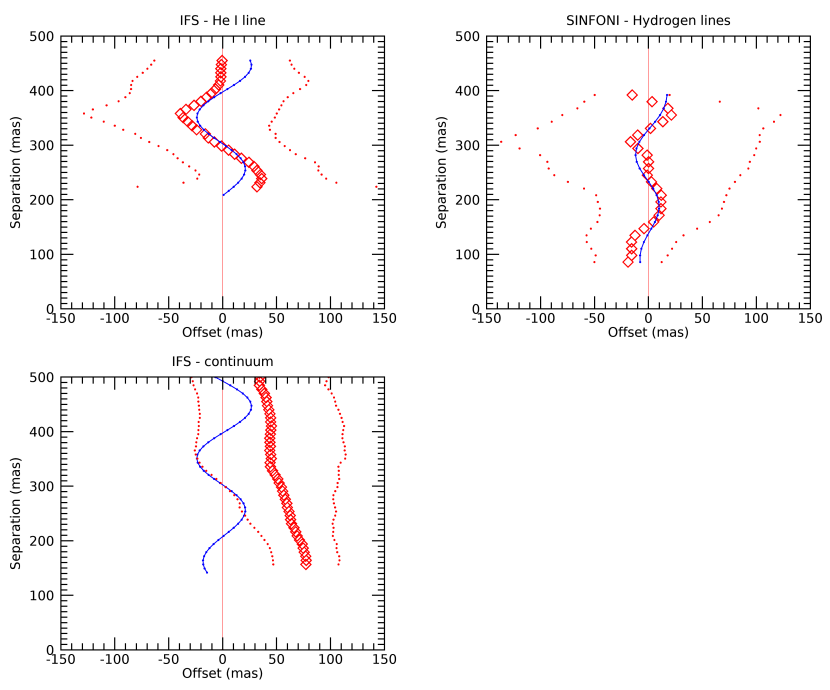

Fig. 7. Peak position of hydrogen and helium lines from SINFONI and SPHERE-IFS data, respectively, as a function of distance from central objects. Dots indicate the sigma of the Gaussian distribution used to obtain the peak position within the slice. The solid blue line is the fit of the wiggle if it is produced by the orbital motion of the jet around the binary system at the center. The blue fit in the three panels is the same, however in the panel showing SINFONI data it is adjusted in order to account for the difference in phase between SPHERE and SINFONI observations and for the different instrument resolution.

wiggling pattern with a projected half-opening angle of $5^{\circ}-6^{\circ}$. It is not surprising to see a wiggle in the jet of young stars: it has been detected in both the other young stars where a jet was observed with SPHERE, namely RY Tau (Garufi et al. 2019) and ZCMa (Antoniucci et al. 2016). Contrary to these previous studies, where the binarity of the central object was assumed from the wiggle of the observed jet, for $\mathrm{R} \mathrm{CrA} \mathrm{we} \mathrm{know} \mathrm{that} \mathrm{the} \mathrm{cen-}$ tral system is a binary star (Sissa et al. 2019). We checked that the rotation period of the binary system (66 days) is in agreement with the phase difference between the two observations, SINFONI and SPHERE-IFS, which are shifted by $\sim$ two months in time. If the observed period of the wiggling (190 mas) is linked to the period of the central binary system, we could then measure the velocity of the jet projected on the sky, which we found to be $\sim 760 \mathrm{~km} \mathrm{~s}^{-1}$. This corresponds to a radial component of the velocity respect to us of $\sim 130 \mathrm{~km} \mathrm{~s}^{-1}$, if we consider that the disk is seen almost edge-on and that the jet is then seen with an inclination of $\sim 10^{\circ}$ on the plane of the sky (see below). We also notice that the wiggling is not visible in the continuum emission. As shown in the bottom panel of Fig. 7, the model obtained from the emission lines (blue solid line), using the same PA measured from the gas lines, does not reproduce the shape of the observation, pointing to the conclusion that the continuum emission does not have a wiggling pattern, even if is appears non-uniform.

Another point coming from the analysis of the SPHEREIFS images in the different channels is that the emission by the gas shown by HeI line is not centered on the continuum emission. This is clear comparing the top and bottom panels of Fig. 1: the HeI emission is not exactly co-located to the continuum emission, appearing shifted to the south direction, almost at the border of the continuum emission, suggesting it is produced in the external layer of the "elongated structure". Moreover, the HeI emission is spatially resolved along the direction perpendicular to the jet elongation, yielding a median jet semi-aperture of $\sim 10 \mathrm{au}$.

\subsection{Geometrical model of the wiggling jet}

We constructed a geometrical model of the wiggling jet of $\mathrm{R} \mathrm{CrA}$, as observed in the HI lines in the SINFONI spectrum. We modeled two observed quantities: the luminosity and the radial velocity maps. The radial velocities were derived by crosscorrelation, and the maps of the intensities and radial velocities of the Hi lines are shown in the top panels of Fig. 8. In order to model these two quantities, we assume that the jet is describing a helix on the surface of a truncated cone, which is seen with an inclination $i$ and position angle PA with respect the sky plane. Since the inclination is very small, the jet velocity on the sky plane is set by the ratio of the separation between the helix pitch and the binary orbital period. In the assumptions that the jet is optically thin, the regions where the spiral crosses the plane of the sky have a greater depth along the line of sight, hence they are seen as blobs in the luminosity image. We identified two consecutive blobs in Fig. 8 as box 1 and box 2. The projected separation between two consecutive blobs on the same side of the jet will then give the helix pitch. As we have seen previously, the jet transverse velocity determined in this way is $760 \mathrm{~km} \mathrm{~s}^{-1}$. The combination of the luminosity and radial velocity maps make it possible to determine all the parameters of the model (see Fig. 8). The PA is the mid-angle of the jet on the sky plane (PA $=67^{\circ}$, in the usual convention starting from north toward east). The ratio between the mean jet radial velocity and the transverse velocity sets the inclination angle at which the helix is seen. We find $i=3.3^{\circ}$, meaning that the jet is seen very close to the sky plane. This suggests that the disk of R Cra is seen almost edge on. In this model, the radial velocity difference between opposite sides of the helix (e.g. box 1 and box 2 in Fig. 8) is due to a projection effect, because they are moving with a different inclination with respect to the line of sight, and then sets the cone aperture angle. The observed difference of $\sim 18 \mathrm{~km} \mathrm{~s}^{-1}$ is reproduced by a semiaperture of the cone of $\sim 1.2^{\circ}$, pointing to a very well collimated jet. Once this angle is fixed, the observed distance between the blobs and the jet axis determines the radius of the base of the cone $r_{\mathrm{B}} \sim 5.3 \mathrm{au}$.

Most interestingly, the model also sets the phase when the jet crossed the binary orbital plane at the epoch of the SINFONI observation (at a distance of 5.3 au from the barycenter of the central binary). Of course this is an idealization of the real jet trajectory because it is unlikely that the jet is launched from this position. However, this can still be useful in understanding the jet geometry with respect to the central binary. We found that this phase is 0.35 , where the zero point is when the binary primary ( $\mathrm{R} \mathrm{CrA} \mathrm{Aa)} \mathrm{is} \mathrm{in} \mathrm{opposition.} \mathrm{For} \mathrm{comparison,} \mathrm{the} \mathrm{binary} \mathrm{model}$ constructed in Sissa et al. (2019) tells us that at the same epoch, the R CrA Aa was at a phase of 0.459. The difference between these two phases is quite small, and may be justified by considering the delay of the jet due to the time required to reach a distance of $5.3 \mathrm{au}$ from the star at a constant speed of $700 \mathrm{~km} \mathrm{~s}^{-1}, 12$ days, that corresponds to a phase difference of 0.18 . Of course, the jet trajectory is likely shorter than one passing through this ideal point. Even if these values contain some uncertainties (e.g. in the exact jet trajectory and velocity and in the exact value of $r_{\mathrm{B}}$ ), they suggest that the jet is actually launched along the direction defined by the two components of the central binary, towards the far side of the primary.

\section{5. [Fe II] and $\mathrm{H}_{2}$ lines}

Table 3 collects radial velocities and full width at half maximum (FWHM) of the spectral lines detected in the SINFONI spectra. 

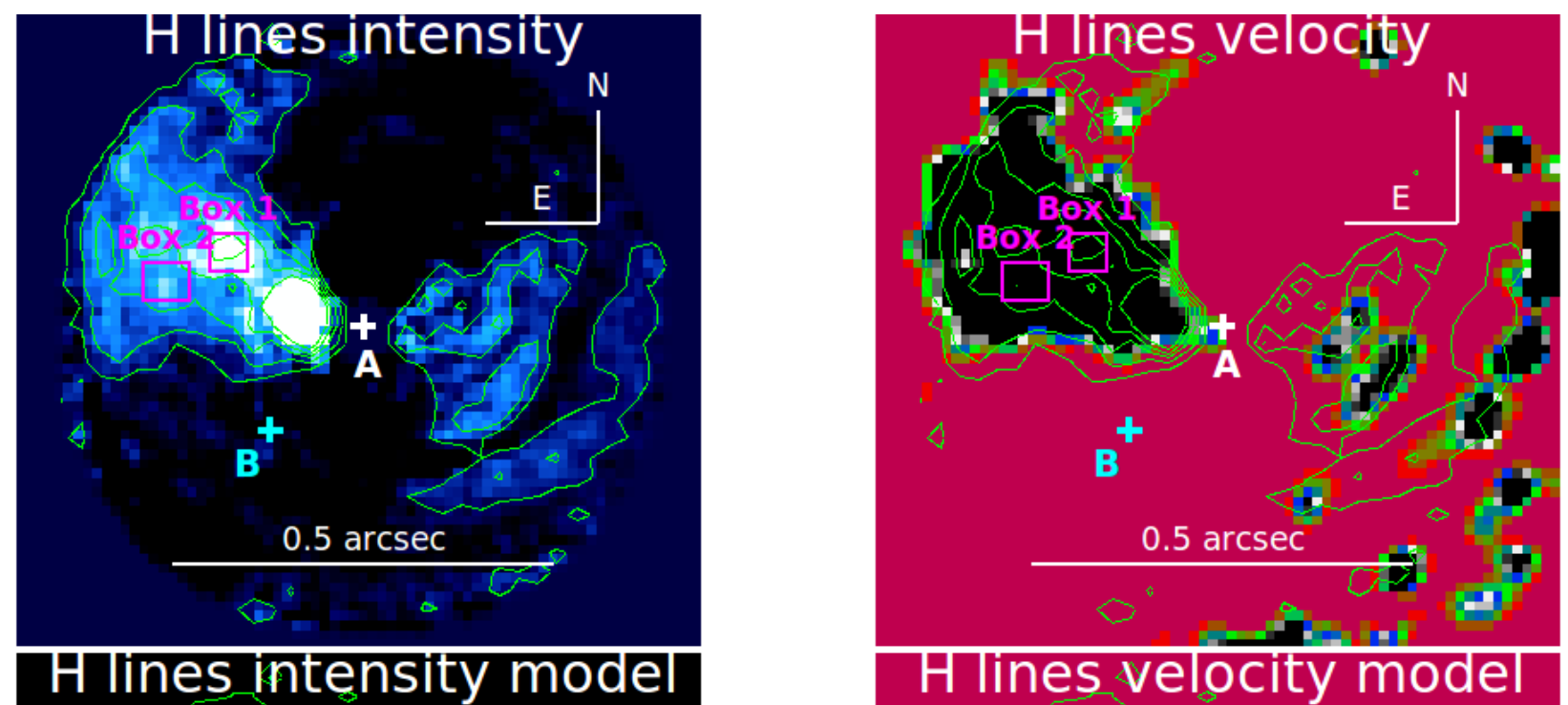

Hines velocity model
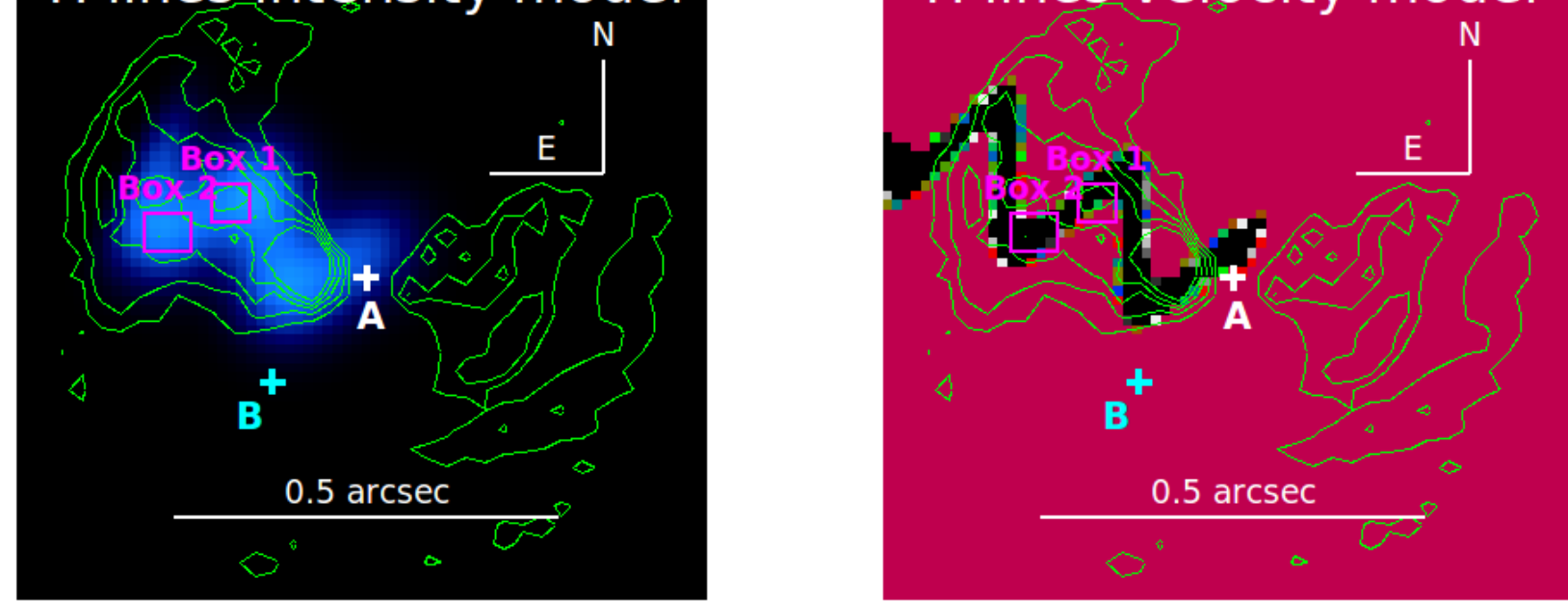

$-1.5$

5.5

9

13

16

19

23

27

Fig. 8. Top panels: intensity and velocity map of HI lines obtained from SINFONI data. The intensity map is obtained by measuring the sum of the intensity of the HI lines in each pixel, and the velocity map is obtained from the cross-correlation function of the image using a correlation mask. Bottom panels: intensity and velocity maps of model. Box 1 and box 2 identify the two consecutive blobs that are used to build the geometrical model of the wiggling, as discussed in the text.

In particular, we collected data for spectra extracted from different regions of the data cube: close to the star, and in the "jet" and dust "cavity" area, as identified in Fig. 3 and in Sect. 2.2. The hydrogen lines are detected in all regions and are usually very broad, consistent, and with a high velocity wind. The [Fe II] are only detected in the dust "cavity" area, tracing an external layer of the "elongated structure". They have low velocities, and are narrow and not resolved in the SINFONI spectrum. The ratio between the intensity of the lines at 1.64 and $1.53 \mu \mathrm{m}$ is a diagnostic of density (Nisini et al. 2005). The observed ratio of about four corresponds to a density of about $10^{4} \mathrm{~cm}^{-3}$. This is an order of magnitude larger than the upper limit obtained from the lack of [Fe II] lines in the "jet" region, but not far from the one estimated using the [S II] lines and the narrow component of the [O I] lines. This suggests that this dust "cavity" region corresponds to the low-velocity component seen in the FEROS spectra (see Table 2).

$\mathrm{H}_{2}$ lines are also clearly detected in the SINFONI spectra, and their emission comes from a region very close to the star, as seen in Fig. 3. Even if an exact quantification is difficult, it suggests that it originated within 230 mas (corresponding to $\sim 35 \mathrm{au}$ from the star). This is within the orbit of the M-dwarf companion, where the circumbinary disk should be. The radial velocities of these lines are in agreement with those derived in the hydrogen lines in the "jet" direction, but the FWHM are much smaller, and the lines are not resolved in the SINFONI spectrum.

The relative intensity of the different $\mathrm{H}_{2}$ lines agree, within $10 \%$, with what was expected from collisional excitation at a temperature of about $15000 \mathrm{~K}$, and it is very different from what was expected from fluorescence models (Black \& van Dishoeck 1987). Namely, mainly low-excitation lines are observed, and the high-excitation lines, which are also expected to be strong due to fluorescence, are absent. The high-temperature gas can be heated by shocks (Shull \& Beckwith 1982) that may be located close to the base of the jet or in the regions where this interacts with the disk. Radial velocities and FWHM of the hydrogen and $\mathrm{H}_{2}$ lines in the "jet" area are in agreement with the values found for the [Fe II] at $7155 \AA$ line in the FEROS spectrum, suggesting that 
Table 3. Radial velocities and FWHM for spectral lines detected in SINFONI spectra.

\begin{tabular}{lcc}
\hline \hline Component & $\begin{array}{c}v_{c} \\
\left(\mathrm{~km} \mathrm{~s}^{-1}\right)\end{array}$ & $\begin{array}{c}F W H M \\
\left(\mathrm{~km} \mathrm{~s}^{-1}\right)\end{array}$ \\
\hline \multicolumn{3}{c}{ Star } \\
\hline $\mathrm{H}$ & $-14.8 \pm 4.0$ & $435 \pm 14$ \\
\hline \multicolumn{3}{c}{ "Jet" } \\
\hline $\mathrm{H}$ & $-39.9 \pm 2.4$ & $393 \pm 7$ \\
$\mathrm{H}$ (box 1) & $-29.7 \pm 3.0$ & $383 \pm 9$ \\
$\mathrm{H}$ (box 2) & $-45.3 \pm 2.7$ & $410 \pm 9$ \\
$\mathrm{H}$ & $+0.7 \pm 4.9$ & $174 \pm 12$ \\
\hline \multicolumn{3}{c}{ "Dust cavity" } \\
\hline$[\mathrm{Fe} \mathrm{II}]$ & $-0.2 \pm 4.5$ & $208 \pm 12$ \\
$\mathrm{H}$ & $-6.6 \pm 8.1$ & $423 \pm 26$ \\
\hline
\end{tabular}

Notes. $v_{c}$ and FWHM were derived by cross-correlation and fitting of the following lines. Hydrogen: brackett series lines from $\mathrm{H}(11-4)$ up to $\mathrm{H}(21-4)$; [FeII]: 1.53347, 1.59948, 1.66377, 1.67688, 1.711132; $\mathrm{H}_{2}: 1.70150,1.71427,1.72902,1.73202$.

this line (that requires densities as high as $\sim 10^{5} \mathrm{~cm}^{-3}$, Nisini et al. 2005) might be also produced in the same post-shock region near the star.

We notice that the $\mathrm{H}_{2}$ lines, which should dominate the $K 1$-band in the IRDIS image, are not detected. To stress this point, we performed the subtraction of the $K 1-K 2$ IRDIS image, as shown in Fig. 2 (bottom panel). On the other hand, fainter $\mathrm{H}_{2}$ lines are detected in the $H$-band SINFONI spectrum. This non-detection of $\mathrm{H}_{2}$ in the $K 1-K 2$ image is essentially due to the fact that SPHERE has been designed for detecting continuum emission, it is not optimized for detecting (extended) line emission. The spectral resolution of SINFONI is two orders of magnitude higher: this makes it possible to detect emission lines with a surface luminosity two orders of magnitude fainter. We verified that the non-detection if the brightest $\mathrm{H}_{2}$ emission line in the $K 1$-band (at $2.12 \mu \mathrm{m},(\mathrm{S}(1)(1-0))$ ) is compatible with the detection of the brightest $\mathrm{H}_{2}$ emission line in the $H$-band (at $1.74 \mu \mathrm{m},(\mathrm{S}(7)(1-0)))$.

\subsection{Spectro-astrometry: information on the verycentral regions}

Some information about the very central region of the system can be obtained using spectro-astrometry (Whelan \& Garcia 2008) on the SINFONI data. We should remind readers that the bulk of the emission from R Cra in the near-IR is due to the warm disk (Sissa et al. 2019). Interferometric observations with the instrument AMBER at VLTI (Kraus et al. 2009) indicate that this emission is offset by about four mas with respect to the barycenter of the system, in the direction of the jet, because selfshadowing of the highly inclined disk only makes it possible to see the far side of the disk. Also, the separation between the central binary components projected along the jet axis is very small (about 0.3-0.4 mas) and can thus be neglected here. Since the scope of spectro-astrometry is to explore regions very close to the center of the system, we used the images obtained by subtraction of a radial profile. The images were corrected for the telluric lines and then cross-correlated with digital masks for $\mathrm{HI}$ and [Fe II] (results for $\mathrm{H} 2$ lines were less clear). The resulting data cube is then made of cross correlation functions - that is, the $z$-coordinate now represents radial velocities. We then rotated these images so that the jet is along the $y$-axis, and collapsed the images along the jet. This is how we obtained bidimensional images, where the axes are radial velocity and offset along the jet with respect to the peak of the continuum emission. We then fitted Gaussians in offset as a function of radial velocity and compared the position of the peak with the value we obtained for the continuum (that, we remind readers, represents the position of the warm disk). Since there is no relevant variation of the position of this peak with velocity, we simply considered the average values. The peak obtained for HI lines is offset by about four mas in the opposite direction to the jet: this indicates that the bulk of the $\mathrm{H}$ emission is caused by material very close to the central binary, likely tracing the accretion. This agrees with the large value of the FWHM. On the other hand, the peak for [Fe II] is offset by about two mas in the direction of the jet, indicating that, in this case, the bulk of the emission comes either from the jet or from regions close to the disk, at about six mas $(\sim 1 \mathrm{au})$ from the star projected along the jet axis.

\section{Proposed scenario and discussion}

In this section, we discuss a scenario where all the features, structures, and properties seen and analyzed in the "elongated structure" are taken into account. The proposed scenario is supported by at least a similar case, the T Tauri star FS Tau B observed by Eislöffel \& Mundt (1998), who imaged the "elongated structure" as the wide outer edges of windblown cavities and the narrower jet flowing inside the cavity in $\mathrm{H} \alpha$.

The schematic picture we consider is obtained from the complementary detailed analysis of the SPHERE (IRDIS and IFS) images and spectra, of the SINFONI data, and of the optical FEROS spectrum. The system appears to be composed of at least two elements: a dusty component seen in scattered light, and a gaseous component, detected in emission atomic lines of forbidden species ([O I], [He I] and [Fe II]) and hydrogen lines. What we have called "elongated structure" until now, which is seen in scattered light in the IFS and IRDIS images, as well as in the SINFONI image, is dominated by continuum emission and is most likely a cavity carved out into the circumstellar environment; the dust on the cavity walls is illuminated by the central binary and scatters light toward us. The jet flowing inside the cavity is detected in the HVC of the optical forbidden lines and in the broad hydrogen lines. The jet is very collimated and shows a wiggling pattern in the hydrogen and [H I] lines, which is in agreement with the orbital period of the central binary. The LVC of the optical forbidden lines suggests the presence of gas moving more slowly, most likely a disk wind. SINFONI data allows us to spatially resolve the [Fe II] emission, which appears to be located at the edge of the cavity, consistent with a layered disk wind as well. This structure is captured in the schematic picture of the R CrA environment that is shown in Fig. 9. The disk, which is illustrated in blue in the figure, is not well detected in the scattered light images, and it is most likely seen edge on. The central binary system (Sissa et al. 2019) is hidden behind the coronagraph, while the wide M-dwarf companion is clearly visible in all the images. The red shell shows the cavity walls, and the green diffuse area the gaseous jet flowing within the cavity.

Differently from FS Tau B (Eislöffel \& Mundt 1998), where the narrower jet flowing inside the cavity points toward the center of the cavity, in the case of R CrA the gaseous jet is not currently pointing toward the center, but rather toward the southern border of the cavity. The misalignment between the axis of the cavity 


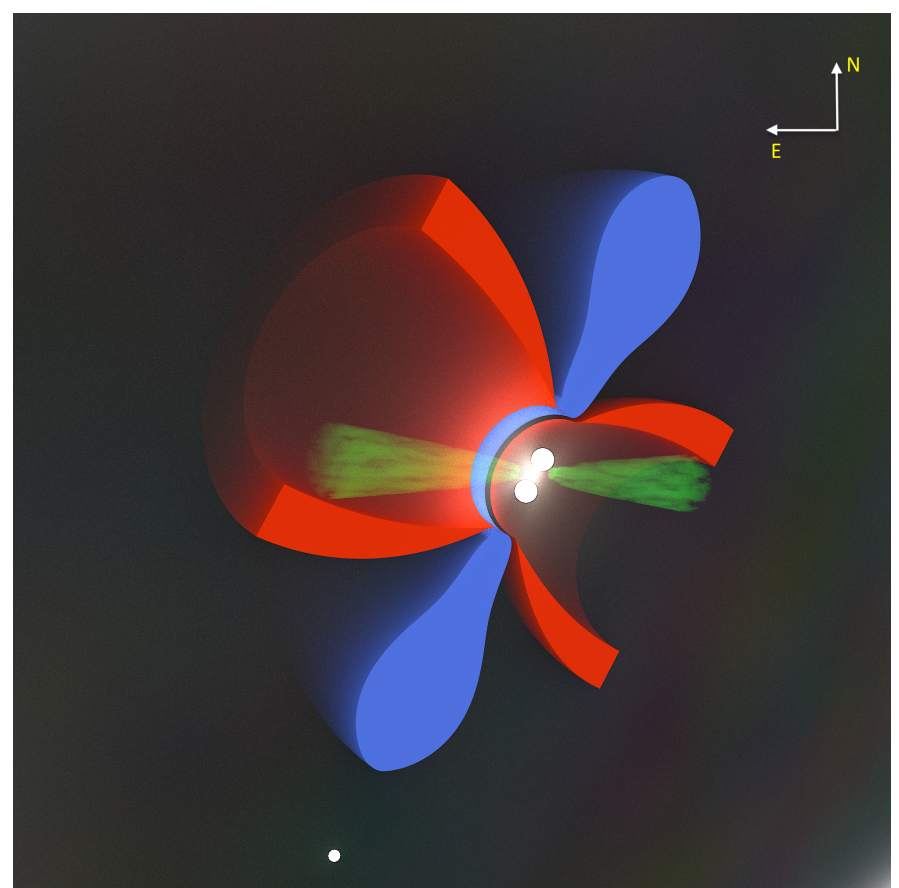

Fig. 9. Sketch of scenario proposed for the "elongated structure" of R CrA. White-filled circles are the three stellar components. The blue region is the circumbinary disk. The green area is the gaseous high velocity jet. The red region is the edge of the dust cavity. The stars of the triple system are represented with the white dots.

and the jet might be due to a temporal variation of the jet direction. In turn this might be attributed on short timescales to a wiggling pattern of the jet related to the central binary orbit, and on longer timescales to a precession motion that tends to change the direction of the jet axis of rotation. This precession motion may be due to the multiplicity of the system, which includes the central binary (Sissa et al. 2019) and the wide companion (Mesa et al. 2019).

This scenario reconciles well with the fact that the environment around $\mathrm{R} \mathrm{CrA}$ is rich in diffuse ambient gas and dust that can be swept-up by powerful jets, as shown by the prominent number of bow shocks, knots, and outflows detected at different wavelengths (e.g. Kumar et al. 2011; Anderson et al. 1997; Groppi et al. 2004, 2007). Moreover, the shock produced by the wind-carved cavity would be responsible, together with the lower mass wide companion, of the emission in X-ray of hot plasma observed with Chandra and XMM-Newton and analyzed by Forbrich et al. (2006). The H2 lines may also form in this shock area.

What can our observations tell us about the launching mechanism for the jet of $\mathrm{R} \mathrm{CrA}$ ? Jets from young stars are usually thought to be launched magneto-centrifugally from disks around them (see e.g. Frank et al. 2014). Favored scenarios consider disk wind (see Pudritz \& Norman 1983) or star-disk magnetosphere (Camenzind 1990), the latter in particular in the X-wind scenario (Shu et al. 2000). The disk-wind scenario can itself be separated into two separate schemes, cold-wind and warmwind (Konigl \& Pudritz 2000). As discussed, for example, by Ferreira et al. (2006), cold winds are generally not considered favorably, because they lead to jets that rotate much faster than usually observed. The main difference between these mechanisms is the launch radius $r_{0}$, which is related to the specific angular momentum of the jet and to the magnetic leverage $\Lambda$, which is the square of the ratio between the Alfvèn radius $r_{\mathrm{A}}$, and $r_{0}: \Lambda=\left(r_{\mathrm{A}} / r_{0}\right)^{2}$. The Alfvèn radius is where the magnetic energy density is equal to the kinetic energy density. Beyond the Alfvèn distance, the field lines lag behind the rotation of their footpoints and are coiled into a spiral (see e.g. Spruit 2010). We then expect that the radius of the base of the truncated cone representing a jet should be connected to the Alfvèn radius, though not necessarily be identical, because the jet may have to traverse many Alfvèn radii before being effectively focused, since its collimation depends not simply on the magnetic lever arm, but also on the poloidal field strength at the disk surface (see Frank et al. 2014). The magnetic leverage is in turn related to the ratio between the jet velocity $V_{\text {jet }}$ and the Keplerian $V_{\text {Kep }}$ at $r_{0}$ by the relation $V_{\text {jet }}=V_{\text {kep }} \sqrt{2 \Lambda-3}$. All these quantities are then related to the launch radius $r_{0}$. As discussed by Frank et al. (2014), typical parameters for jets around T Tau stars are compatible with $r_{0}$ of the order of a few stellar radii. The large value of $V_{\text {Kep }}$ at such small separation implies a small value for the magnetic leverage factor $(\sim 5-10)$ and slow jet rotation $\left(\sim 10 \mathrm{~km} \mathrm{~s}^{-1}\right)$. These are compatible with the very few detections of jet rotation (see e.g. the case of HH212, Lee et al. 2017, or the more questioned case of DG Tau: Bacciotti et al. 2002; White et al. 2014).

Since R CrA is a binary with a separation of $a \sim 0.56$ au, which is $\sim 119 R_{\odot}$, it is possible that its jet might be quite peculiar. The geometrical model discussed in the previous section suggests that the jet is launched close to the primary. However, the presence of a massive enough disk around it is not obvious, because we expect such a disk to be truncated at about a third of the Hill radius, which is at about $20 R_{\odot}$ from the star. Since it is likely that the magnetic field of the whole binary system is locked to the binary orbit, the jet orientation would be determined by the orbital phase. On the other hand, the jet velocity is much larger than the escape velocity from the binary. As a consequence, we might expect that the jet describes a helix that may be similar to what is observed. If it exists, such a jet would have properties similar to those observed for other very young stars; in particular, it would be slowly rotating (at most a few tens of $\mathrm{km} \mathrm{s}{ }^{-1}$ ), meaning below the spectral resolution offered by SINFONI.

As another option, we might perhaps consider the case of a jet that is launched at the inner edge of the circumbinary disk, which is with $r_{0}$ of the order of $1.5 a \sim 0.84$ au. Such a jet would have peculiar characteristics. Given the high specific angular momentum of material at this location, we would expect a rapidly rotating jet. The relation between $r_{0}$ and the jet rotational velocity $V_{\text {rot }}$ is obtained considering that $r_{0}=0.05 \times(2 \times$ $\left.\left(l_{J} / 10\right) /\left(V_{\text {jet }} / 115\right)\right)^{2} \times(M / 0.25)^{1 / 3}$ (Lee et al. 2017), where $M$ is the stellar mass and $l_{J}$ is the jet specific angular momentum (in au $\times \mathrm{km} \mathrm{s}^{-1}$ ). Assuming typical values appropriate for the case of R CrA (jet size $\sim 25$ mas, $\sim 4$ au; jet velocity $V_{\text {jet }}=700 \mathrm{~km}$ $\mathrm{s}^{-1}$; stellar mass $=3.0 M_{\odot}$ ), we obtain $V_{\text {rot }} \sim 180 \mathrm{~km} \mathrm{~s}^{-1}$. This is much larger than typically observed in $\mathrm{T}$ Tau stars, but not incompatible with the value we obtain for the Half Width Half Maximum of the $\mathrm{H}$ lines in direction of the jet (and of blobs 1 and 2) that is $\sim 400 \mathrm{~km} \mathrm{~s}^{-1}$. This is about twice the spectral resolution of SINFONI, and indeed the $\mathrm{H}$ lines are clearly much broader than the [Fe II] or $\mathrm{H}_{2}$ lines (that are not seen in the jet).

Another peculiar property expected for such a jet is the large leverage factor $\Lambda \sim 44$. This would imply $\xi=1 /[2(\Lambda-$ $1)]=0.01$ and $r_{\mathrm{A}}=5.5 \mathrm{au}$. $\xi$ is related to the ratio between the mass-loss rate through the jet and the accretion mass rate, $\xi \sim \dot{M}_{\text {jet }} / \dot{M}_{\text {acc }}$. Hence a low value of $\xi$ implies a low value for 
$\dot{M}_{\text {jet }}$, which in turn might explain the low density of the jet of $\mathrm{R} \mathrm{CrA}$. The Alfvèn radius would be actually similar to the radius of the base of the cone $r_{\mathrm{B}}=5.3$ au derived in our geometrical model. The high value of $\xi$ is compatible with a well collimated jet (see e.g. Garcia et al. 2001). Finally, it should be noted that such a large magnetic leverage factor would be more compatible with a cold-wind jet scenario.

In summary, the nature of the jet seen in R CrA is still not clear. Observations are compatible both with the usual warmdisk or X-wind scenarios, but a cold-wind scenario is also possible, related to the peculiar fact that $\mathrm{R} \mathrm{CrA}$ is a binary with a separation of a few tens of stellar radii. The two cases might be distinguished by a more accurate estimate of the specific angular momentum of the jet, which might be possible using ALMA (see e.g. Lee et al. 2017) for example.

\section{Conclusions}

Taking advantage of the complementary information provided by optical spectroscopy data (acquired with FEROS) and adaptive optics images in the near infrared obtained with SPHERE and SINFONI, we investigated the extended structure seen around the Herbig Ae/Be star R CrA. R CrA is a very interesting system, not only because it is bright, quite massive, and very young, but also because it is a triple system with a central binary whose separation is of the order of a few tens of the radii of the individual components. This separation might be critical to the survival of circumstellar disks that are considered in the most popular scenarios of magneto-centrifugally launched jets. The vicinity of this star, together with the spatial resolution offered by complementary instrumentation allowed us to investigate in great detail the extended structures seen around $\mathrm{R} \mathrm{CrA}$. The data reveal a complex overall structure composed by three components: a collimated jet, a wide cavity, and a shock region close to the central system.

A well collimated gaseous jet has been detected in the hydrogen (with SINFONI) and [He I] (with SPHERE) emission lines. It shows a wiggling pattern that is consistent with the period of the binary system. We implemented a geometrical model to reproduce this wiggling pattern, showing that observations may be reproduced by a high velocity $\left(\sim 770 \mathrm{~km} \mathrm{~s}^{-1}\right)$ jet inclined by $3.3^{\circ}$ toward the observer, which describes a helix on the surface of a cone. The HVC seen in the optical forbidden lines with FEROS is most likely associated with this fast-collimated jet that is flowing inside a cavity carved in the interstellar medium.

The wide cavity is seen in the continuum emission of the IRDIS and IFS SPHERE data. It shows as a non-uniform extended structure that extends up to $400 \mathrm{au}$ in the N-E direction, and as wide as $\sim 30^{\circ}$. The cavity walls are seen in scattered light. The fast-collimated jet appears to be pointing toward the southern side of the cavity, meaning it is not oriented toward the center of the wide cavity, most likely due to a precession motion. [Fe II] emission is detected along the wall of the cavity. This emission might be attributed to a slower moving wind, most likely a disk wind, that is also producing the LVC seen in the optical forbidden lines.

The third component is a shock region, close to the central star, where $\mathrm{H}_{2}$ (observed with SINFONI) and possibly the [Fe II] (observed with FEROS) at $7155 \AA$ are produced at higher density. The velocities of these lines and their FWHM are in between the velocities of the HVC and LVC.

$\mathrm{R}$ CrA represents a very interesting object, because it allows us to study the structure of jets around Herbig stars, which is not yet well understood. The overall scenario agrees closely with general expectations for magneto-centrifugally launched jets: however, the fact that the star is actually a triple system likely makes the scenario more complex. Given the relevance of this particular object in our understanding of jets from very young stars, more observations would be welcomed to confirm our findings. Namely, a spectroscopic follow-up both with high-resolution spectrographs (in the optical and near-infrared) and with diffraction-limited integral-field spectrographs (e.g. SPHERE, MUSE, and ERIS) may better constrain the regions where the different lines emit, and the kinematic model used to interpret the fast collimated jet. Finally, the launch region of the jet might possibly be established using high-spatial and spectralresolution observations of the jet with ALMA for example, which may be used to determine the jet rotational velocity.

Acknowledgements. E.R. is supported by the European Union's Horizon 2020 research and innovation programme under the Marie Skłodowska-Curie grant agreement No 664931. This work has been supported by the project PRIN INAF 2016 The Cradle of Life - GENESIS-SKA (General Conditions in Early Planetary Systems for the rise of life with SKA) and by the "Progetti Premiali" funding scheme of the Italian Ministry of Education, University, and Research. Programme National de Planétologie (PNP) and the Programme National de Physique Stellaire (PNPS) of CNRS-INSU. This work has also been supported by a grant from the French Labex OSUG2020 (Investissements d'avenir - ANR10 LABX56). The project is supported by CNRS, by the Agence Nationale de la Recherche (ANR-14-CE33-0018). This work has made use of the SPHERE Data Centre, jointly operated by OSUG/IPAG (Grenoble), PYTHEAS/LAM CeSAM (Marseille), OCA/Lagrange (Nice), Observatoire de Paris/LESIA (Paris), and Observatoire de Lyon/CRAL. We thank P. Delorme and E. Lagadec (SPHERE Data Centre) for their efficient help during the data reduction process. SPHERE is an instrument designed and built by a consortium consisting of IPAG (Grenoble, France), MPIA (Heidelberg, Germany), LAM (Marseille, France), LESIA (Paris, France), Laboratoire Lagrange (Nice, France), INAF Osservatorio Astronomico di Padova (Italy), Observatoire de Geneve (Switzerland), ETH Zurich (Switzerland), NOVA (Netherlands), ONERA (France) and ASTRON (Netherlands) in collaboration with ESO. SPHERE was funded by ESO, with additional contributions from CNRS (France), MPIA (Germany), INAF (Italy), FINES (Switzerland) and NOVA (Netherlands). SPHERE also received funding from the European Commission Sixth and Seventh Framework Programmes as part of the Optical Infrared Coordination Network for Astronomy (OPTICON) under grant number RII3-Ct-2004-001566 for FP6 (2004-2008), grant number 226604 for FP7 (2009-2012), and grant number 312430 for FP7 (2013-2016). G.v.d.P. acknowledges funding from the ANR of France under contract number ANR-16-CE31-0013 (Planet-Forming-Disks).

\section{References}

Acke, B., van den Ancker, M. E., \& Dullemond, C. P. 2005, A\&A, 436, 209 Alcalá, J. M., Manara, C. F., Natta, A., et al. 2017, A\&A, 600, A20 Anderson, I. M., Harju, J., Knee, L. B. G., \& Haikala, L. K. 1997, A\&A, 321, 575

Antoniucci, S., Podio, L., Nisini, B., et al. 2016, A\&A, 593, L13

Bacciotti, F., Ray, T. P., Mundt, R., Eislöffel, J., \& Solf, J. 2002, ApJ, 576, 222

Banzatti, A., Pascucci, I., Edwards, S., et al. 2019, ApJ, 870, 76

Bellingham, J. G., \& Rossano, G. S. 1980, AJ, 85, 555

Benisty, M., Stolker, T., Pohl, A., et al. 2017, A\&A, 597, A42

Beuzit, J. L., Vigan, A., Mouillet, D., et al. 2019, A\&A, 631, A155

Bibo, E. A., The, P. S., \& Dawanas, D. N. 1992, A\&A, 260, 293

Black, J. H., \& van Dishoeck, E. F. 1987, ApJ, 322, 412

Bonnet, H., Conzelmann, R., Delabre, B., et al. 2004, SPIE Conf. Ser., 5490, 130 Camenzind, M. 1990, Rev. Mod. Astron., 3, 234

Chauvin, G., Desidera, S., Lagrange, A. M., et al. 2017, in SF2A-2017: Proceedings of the Annual meeting of the French Society of A\&A

Chen, H., Grenfell, T. G., Myers, P. C., \& Hughes, J. D. 1997, ApJ, 478, 295

Claudi, R. U., Turatto, M., Gratton, R. G., et al. 2008, SPIE Conf. Ser., 7014 70143E

Cohen, M., \& Kuhi, L. V. 1979, ApJS, 41, 743

Cugno, G., Quanz, S. P., Launhardt, R., et al. 2019, A\&A, 624, A29

Devine, D., Grady, C. A., Kimble, R. A., et al. 2000, ApJ, 542, L115

Dohlen, K., Langlois, M., Saisse, M., et al. 2008, SPIE Conf. Ser., 7014, 70143L

Edwards, S. 2007, Star-Disk Interaction in Young Stars, eds. J. Bouvier, \& I.

Appenzeller, IAU Symp., 243, 171

Edwards, S., Cabrit, S., Strom, S. E., et al. 1987, ApJ, 321, 473 
Eisenhauer, F., Abuter, R., Bickert, K., et al. 2003, SPIE Conf. Ser., 4841, 1548

Eislöffel, J., \& Mundt, R. 1998, AJ, 115, 1554

Ellerbroek, L. E., Podio, L., Dougados, C., et al. 2014, A\&A, 563, A87

Fairlamb, J. R., Oudmaijer, R. D., Mendigutia, I., Ilee, J. D., \& van den Ancker, M. E. 2017, MNRAS, 464, 4721

Fedele, D., Carney, M., Hogerheijde, M., et al. 2017, A\&A, 600, A72

Fedele, D., Tazzari, M., Booth, R., et al. 2018, A\&A, 610, A24

Feldt, M., Olofsson, J., Boccaletti, A., et al. 2017, A\&A, 601, A7

Ferreira, J., Dougados, C., \& Cabrit, S. 2006, A\&A, 453, 785

Finkenzeller, U., \& Mundt, R. 1984, A\&AS, 55, 109

Forbrich, J., Preibisch, T., \& Menten, K. M. 2006, A\&A, 446, 155

Frank, A., Ray, T. P., Cabrit, S., et al. 2014, in Protostars and Planets VI, eds. H. Beuther, R. S. Klessen, C. P. Dullemond, \& T. Henning (Tucson, AZ: University of Arizona Press), 451

Garcia, P. J. V., Ferreira, J., Cabrit, S., \& Binette, L. 2001, A\&A, 377, 589

Garcia Lopez, R., Natta, A., Testi, L., \& Habart, E. 2006, A\&A, 459, 837

Garcia Lopez, R., Nisini, B., Eislöffel, J., et al. 2010, A\&A, 511, A5

Garufi, A., Podio, L., Bacciotti, F., et al. 2019, A\&A, 628, A68

Ginski, C., Stolker, T., Pinilla, P., et al. 2016, A\&A, 595, A112

Grady, C. A., Devine, D., Woodgate, B., et al. 2000, ApJ, 544, 895

Grady, C., Woodgate, B., Kimble, R., et al. 2003, IAU Symp., 221, 71

Grady, C. A., Woodgate, B., Torres, C. A. O., et al. 2004, ApJ, 608, 809

Grady, C. A., Hamaguchi, K., Schneider, G., et al. 2010, ApJ, 719, 1565

Graham, J. A. 1993, PASP, 105, 561

Groppi, C. E., Kulesa, C., Walker, C., \& Martin, C. L. 2004, ApJ, 612, 946

Groppi, C. E., Hunter, T. R., Blundell, R., \& Sandell, G. 2007, ApJ, 670, 489

Gullikson, K., Dodson-Robinson, S., \& Kraus, A. 2014, AJ, 148, 53

Hamann, F. 1994, ApJS, 93, 485

Hartigan, P., \& Graham, J. A. 1987, AJ, 93, 913

Hartigan, P., Morse, J. A., \& Raymond, J. 1994, ApJ, 436, 125

Hartigan, P., Edwards, S., \& Ghandour, L. 1995, ApJ, 452, 736

Herbig, G. H. 1960, ApJS, 4, 337

Hillenbrand, L. A., Strom, S. E., Vrba, F. J., \& Keene, J. 1992, ApJ, 397, 613

Huang, J., Andrews, S. M., Cleeves, L. I., et al. 2018, ApJ, 852, 122

Hubrig, S., Jarvinen, S. P., Schöller, M., et al. 2019, ASP Conf. Ser., 518, 18

Konigl, A., \& Pudritz, R. E. 2000, in Protostars and Planets IV, eds. V. Mannings, A. P. Boss, \& S. S. Russell (Tucson, AZ: University of Arizona Press), 759

Kraus, S., Hofmann, K. H., Malbet, F., et al. 2009, A\&A, 508, 787

Kumar, M. S. N., Sharma, S., Davis, C. J., Borissova, J., \& Grave, J. M. C. 2011 A\&A, 533, A137

Kwan, J., \& Tademaru, E. 1988, ApJ, 332, L41

Lee, C.-F., Ho, P. T. P., Li, Z.-Y., et al. 2017, Nat. Astron., 1, 0152

Levreault, R. M. 1988, ApJS, 67, 283

Ligi, R., Vigan, A., Gratton, R., et al. 2018, MNRAS, 473, 1774

Macintosh, B., Graham, J. R., Ingraham, P., et al. 2014, Proc. Natl. Acad. Sci., 111,12661

Maire, A. L., Stolker, T., Messina, S., et al. 2017, A\&A, 601, A134

Malfait, K., Bogaert, E., \& Waelkens, C. 1998, A\&A, 331, 211

Marois, C., Lafrenière, D., Doyon, R., Macintosh, B., \& Nadeau, D. 2006, ApJ, 641,556
Melnikov, S., Woitas, J., Eislöffel, J., et al. 2008, A\&A, 483, 199

Mendigutía, I., Mora, A., Montesinos, B., et al. 2012, A\&A, 543, A59

Mesa, D., Gratton, R., Zurlo, A., et al. 2015, A\&A, 576, A121

Mesa, D., Bonnefoy, M., Gratton, R., et al. 2019, A\&A, 624, A4

Milli, J., Mouillet, D., Fusco, T., et al. 2017, arXiv e-prints [arXiv:1710.05417]

Natta, A., Palla, F., Butner, H. M., Evans, N. J., II., \& Harvey, P. M. 1993, ApJ, 406, 674

Natta, A., Testi, L., Alcalá, J. M., et al. 2014, A\&A, 569, A5

Neuhäuser, R., \& Forbrich, J. 2008, Handbook of Star Forming Regions, ed. B. Reipurth (San Francisco: ASP), 5, 735

Nisini, B., Bacciotti, F., Giannini, T., et al. 2005, A\&A, 441, 159

Nisini, B., Antoniucci, S., Alcalá, J. M., et al. 2018, A\&A, 609, A87

Osterbrock, D. E. 1989, Astrophysics of gaseous nebulae and active galactic nuclei (Sausalito, CA: University Science Books)

Pérez, L. M., Carpenter, J. M., Andrews, S. M., et al. 2016, Science, 353, 1519

Perrot, C., Boccaletti, A., Pantin, E., et al. 2016, A\&A, 590, L7

Podio, L., Bacciotti, F., Nisini, B., et al. 2006, A\&A, 456, 189

Pohl, A., Sissa, E., Langlois, M., et al. 2017, A\&A, 605, A34

Pojmanski, G. 1997, Acta Astron., 47, 467

Proxauf, B., Öttl, S., \& Kimeswenger, S. 2014, A\&A, 561, A10

Pudritz, R. E., \& Norman, C. A. 1983, ApJ, 274, 677

Ray, T., Dougados, C., Bacciotti, F., Eislöffel, J., \& Chrysostomou, A. 2007, in Protostars and Planets V, eds. B. Reipurth, D. Jewitt, \& K. Keil (Tucson, AZ: University of Arizona Press), 231

Rigliaco, E., Pascucci, I., Gorti, U., Edwards, S., \& Hollenbach, D. 2013, ApJ, 772,60

Shu, F. H., Najita, J. R., Shang, H., \& Li, Z. Y. 2000, in Protostars and Planets IV, eds. V. Mannings, A. P. Boss, \& S. S. Russell (Tucson, AZ: University of Arizona Press), 789

Shull, J. M., \& Beckwith, S. 1982, ARA\&A, 20, 163

Sissa, E., Gratton, R., Garufi, A., et al. 2018, A\&A, 619, A160

Sissa, E., Gratton, R., Alcala, J. M., et al. 2019, A\&A, 630, A132

Soummer, R., Pueyo, L., \& Larkin, J. 2012, ApJ, 755, L28

Spruit, H. C. 2010, The Jet Paradigm, ed. T. Belloni (Berlin: Springer), 794, 233

Strom, S. E., Strom, K. M., Yost, J., Carrasco, L., \& Grasdalen, G. 1972, ApJ, 173,353

Takami, M., Bailey, J., \& Chrysostomou, A. 2003, A\&A, 397, 675

Taylor, K. N. R., \& Storey, J. W. V. 1984, MNRAS, 209, 5 P

van der Marel, N., Van Dishoeck, E. F., Bruderer, S., et al. 2013, Science, 340 , 1199

van der Plas, G., van den Ancker, M. E., Waters, L. B. F. M., \& Dominik, C. 2015, A\&A, 574, A75

Vigan, A., Moutou, C., Langlois, M., et al. 2010, MNRAS, 407, 71

Walker, C. K., Lada, C. J., \& Hartigan, P. 1984, BAAS, 16, 998

Wang, H., Mundt, R., Henning, T., \& Apai, D. 2004, ApJ, 617, 1191

Whelan, E., \& Garcia, P. 2008, Jets from Young Stars II, eds. F. Bacciotti, L. Testi, \& E. Whelan, 742, 123

White, M. C., McGregor, P. J., Bicknell, G. V., Salmeron, R., \& Beck, T. L. 2014, MNRAS, 441, 1681 